\title{
Toroidal Imploding Detonation Wave Initiator for Pulse Detonation Engines
}

\author{
S. I. Jackson* $*$ and J. E. Shepherd \\ California Institute of Technology, Pasadena, California 91125
}

DOI: $10.2514 / 1.24662$

\begin{abstract}
Imploding toroidal detonation waves were used to initiate detonations in propane-air and ethylene-air mixtures inside of a tube. The imploding wave was generated by an initiator consisting of an array of channels filled with acetylene-oxygen gas and ignited with a single spark. The initiator was designed as a low-drag initiator tube for use with pulse detonation engines. To detonate hydrocarbon-air mixtures, the initiator was overfilled so that some acetylene oxygen spilled into the tube. The overfill amount required to detonate propane air was less than $2 \%$ of the volume of the 1-m-long, 76-mm-diam tube. The energy necessary to create an implosion strong enough to detonate propane-air mixtures was estimated to be $13 \%$ more than that used by a typical initiator tube, although the initiator was also estimated to use less oxygen. Images and pressure traces show a regular, repeatable imploding wave that generates focal pressures in excess of 6 times the Chapman-Jouguet pressure. A theoretical analysis of the imploding toroidal wave performed using Whitham's method was found to agree well with experimental data and showed that, unlike imploding cylindrical and spherical geometries, imploding toroids initially experience a period of diffraction before wave focusing occurs. A nonreacting numerical simulation was used to assist in the interpretation of the experimental data.
\end{abstract}

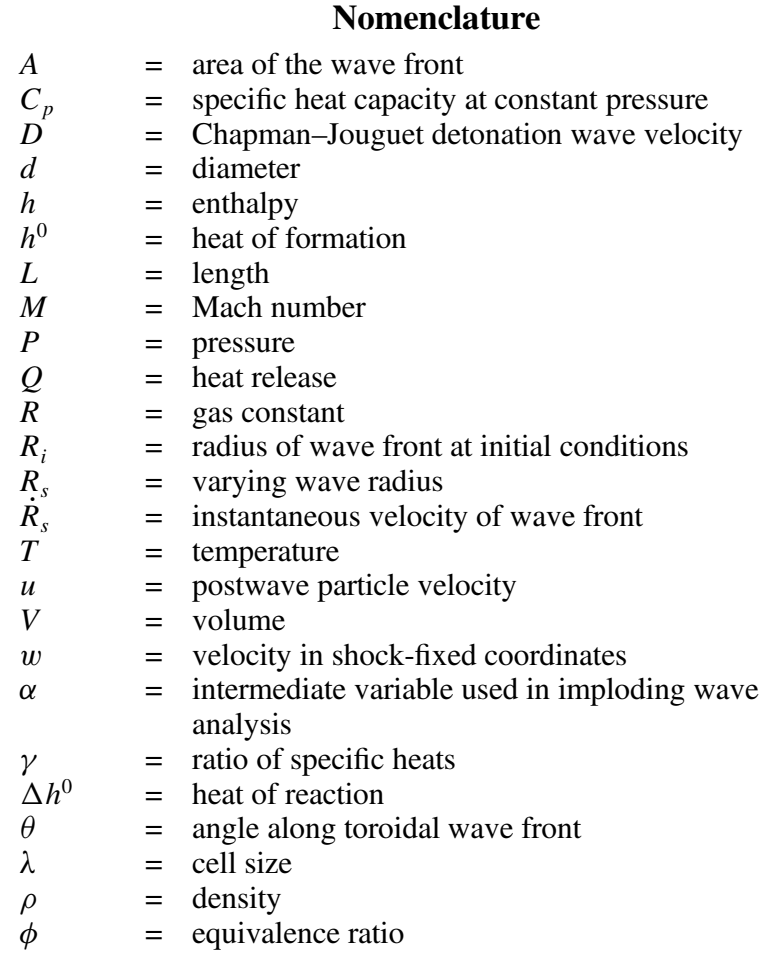

Subscripts

$\mathrm{CJ}=$ Chapman-Jouguet detonation state

$d \quad=$ initiator tube or driver property

Received 17 April 2006; revision received 20 October 2006; accepted for publication 20 October 2006. Copyright $@ 2006$ by California Institute of Technology. Published by the American Institute of Aeronautics and Astronautics, Inc., with permission. Copies of this paper may be made for personal or internal use, on condition that the copier pay the $\$ 10.00$ per-copy fee to the Copyright Clearance Center, Inc., 222 Rosewood Drive, Danvers, MA 01923; include the code $\$ 10.00$ in correspondence with the CCC.

*Postdoctoral Scholar, Shock Detonation Physics, Los Alamos National Laboratory, Los Alamos, NM 87545.

†Professor, Aeronautics, Caltech MC 105-50, Pasadena, CA 91125. Member AIAA.

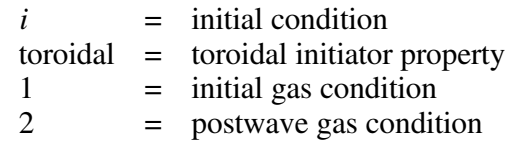

\section{Introduction}

$\mathbf{E}$ FFICIENT detonation initiation of hydrocarbon-air (HC-air) mixtures is critical to the success of pulse detonation engine (PDE) technology [1]. Existing engines [2,3] rely on either deflagration-to-detonation transition (DDT) or initiator tubes to detonate insensitive mixtures such as JP10-air or $\mathrm{C}_{3} \mathrm{H}_{8}$-air. Both of these initiation schemes have drawbacks.

In DDT, induced flow turbulence is used to accelerate a weak flame to detonation [4]. Although the DDT process permits detonation initiation with low-energy sparks, it requires impractically long distances $[\underline{5}, 6]$ in the HC-air mixtures. The presence of turbulence-inducing obstacles in the tube can also incur significant flow losses [6].

Initiator tubes are more appealing as they are able to detonate $\mathrm{HC}$ air mixtures over shorter distances. An initiator tube is a tube filled with a sensitive (or easily detonable) mixture that is connected to a larger-diameter tube filled with a less-sensitive mixture such as HCair. Low-energy ignition and DDT are used to create a detonation in the sensitive mixture over a short distance. That detonation then propagates out into the larger tube and creates a decaying blast wave, which will develop into a detonation if the postshock flow is of sufficient overpressure and duration $[\underline{7}, 8]$. To the frustration of airbreathing PDE designers, sensitization of the initiator tube mixture is best accomplished by the addition of oxygen, requiring tanks of this gas to be carried on any PDE system. To date, the performance of other sensitizing additives has been found lacking [5]. Thus, as initiator tubes remain prevalent as PDE initiators, efforts to decrease their oxygen dependence have focused on increasing the transmission efficiency of the initiator tube through modification of its geometry $[9,10]$. In this paper, work in this area is briefly summarized and followed by a detailed description of initiator tube developments using shock wave focusing at the California Institute of Technology.

In shock focusing, a collapsing shock or detonation wave generates a high-pressure and high-temperature focal region by adiabatically compressing shocked gas as it flows into an everdecreasing area [11-18]. This compression increases the 
postdetonation wave pressure higher than the Chapman-Jouguet (CJ) pressure, resulting in an increasingly overdriven detonation wave.

The overdrive can be used to increase the overpressure of the shock transmitted from the initiator section into the main tube of a PDE and has been shown to increase the transmission efficiency [19]. This technique is dependent only on the geometry of the initiator wave and provides the means to dramatically increase initiator effectiveness and reduce the amount of initiator gas used.

Murray et al. [19] experimentally noted an increase in transmission efficiency when the detonation in the initiator was transmitted into the detonation tube through an annular orifice. With simulations, they reasoned that the annular orifice generated an imploding toroidal wave in the test section. The high pressure and temperature at the focus of the imploding toroid created a region of high-energy density that was capable of evolving into a selfsustaining detonation wave. In particular, they noted that the inclusion of the annular orifice allowed successful detonation transmission for tubes with diameters 2.2 times smaller than tubes with simple circular orifices.

Improving on this concept, a toroidal detonation initiator was developed $[10,20,21]$ to successfully detonate HC-air mixtures in a 1-m-long tube with an imploding toroidal wave that was propagated into the $\mathrm{HC}$-air mixture from the tube walls. To generate the imploding wave, the toroidal initiator used a single spark plug and a small amount of acetylene-oxygen gas.

More recent numerical [22] and experimental [23] work investigated the ability of imploding toroidal shock waves (rather than imploding detonation waves) to initiate detonations in HCoxygen mixtures that are highly diluted with nitrogen. Simulations by $\mathrm{Li}$ and Kailasanath [22] of an imploding toroidal shock wave driven by jets of air or fuel determined that an imploding annular jet with a Mach number of unity, a pressure of $0.2 \mathrm{MPa}$, and a temperature of $250 \mathrm{~K}$ (corresponding to a total pressure and temperature of $0.38 \mathrm{MPa}$ and $470 \mathrm{~K}$, respectively) was able to initiate a detonation in a stoichiometric ethylene-air mixture inside of a tube. However, subsequent experimental work [23] with a design similar to that specified by $\mathrm{Li}$ and Kailasanath [2] was unable to initiate ethylene-air mixtures, even using sonic jets with total pressures and temperatures in excess $(1.68 \mathrm{MPa}$ and $790 \mathrm{~K})$ of those used in the numerical simulations [22].

Through detailed analysis of the implosion process, simulations at Ohio State $[24,25]$ have shown that the reflection of the primary explosion from the contact surface, which separates the gas initially in the tube from the injected gas driving the implosion, creates a secondary implosion that is responsible for the creation of the high pressures and temperatures that lead to detonation initiation in this geometry.

de Witt et al. [26] have also detonated ethylene-air mixtures from a $150 \mathrm{~mJ}$ spark in a distance of $1.47 \mathrm{~m}$ with shock wave focusing. First, they used orifice plates to accelerate a flame in a tube and promote shock formation. Once a shock of sufficient strength was developed, it was diffracted around a cone-shaped obstacle, creating an imploding cylindrical wave downstream of the obstacle that was able to detonate the mixture.

\section{Initiator Design}

The toroidal initiator was created by mapping the geometry of a planar wave initiator [27] to the surface of an aluminum cylinder such that the exit of each channel lies on a circle with the channels exhausting inward. (A planar wave initiator is used to create a planar detonation front from a single ignition point and an array of channels.) With this mapping, the metal substrate of the planar initiator became a cylinder with the channels milled on its outer surface (Fig. 1). This inner cylinder or sleeve had an outer diameter of $101.0 \mathrm{~mm}$ and an inner diameter of $76.2 \mathrm{~mm}$, which formed the main tube. A second, 37.97-cm-long, aluminum cylinder with inner and outer diameters of $100.9 \mathrm{~mm}$ and $139.7 \mathrm{~mm}$, respectively, was used as the outer sleeve shown in Fig. 1 to seal the open surface of channels machined on the inner sleeve. This seal was accomplished by an
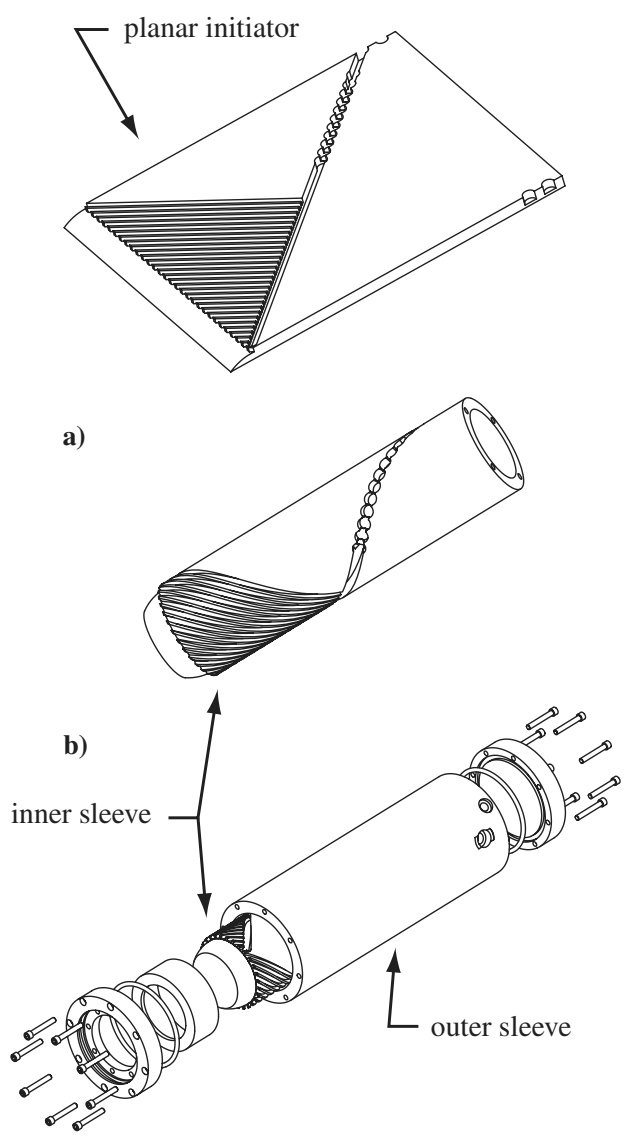

c)

Fig. 1 The planar geometry a) is mapped to a cylindrical geometry b) to create the inner sleeve. The inner sleeve is then inserted into the outer sleeve using shrink-fitting techniques c).

interference fit, as the outer diameter of the inner sleeve was larger than the inner diameter of the outer sleeve by $0.1 \mathrm{~mm}$. Before assembly, the inner sleeve was cooled in liquid nitrogen and the outer sleeve was kept at room temperature. The resulting thermal contraction decreased the outer diameter of the inner sleeve so that it could be inserted into the outer sleeve. Once the temperature of the two pieces had equilibrated, the residual stress held the two sleeves together and maintained a tight seal at the interface.

A ramp was placed at the exhaust of the secondary channels to focus the emerging wavelets inward toward the axis of the device. This design allows the initiator to be incorporated into the walls of a PDE, minimizing the drag losses by not obstructing the main tube flow path in PDE applications.

Two toroidal initiators (Fig. 2) were constructed that were identical in all aspects except for their channel geometries. A static initiator was designed and constructed as a prototype and a dynamic initiator was constructed for PDE applications, which require sensitive driver gas injection into the small channels [27]. All results presented were obtained with the dynamic initiator, which shaped the implosion from six series of square channels located on the inner cylinder. The dimensions of each channel series are shown in Table 1. For visualization purposes, the channel geometry of the dynamic toroidal initiator has been mapped to a planar surface in Fig. 3. Detailed design information on both initiators, including drawings, a parts description, assembly instructions, and an example calculation of the shrink-fitting theory, is available in other work $[21,28]$.

During operation, the initiator channels were filled with a detonable mixture and a flame was ignited by discharging a $46 \mathrm{~mJ}$ spark near the start of the primary channel. The flame front then underwent DDT due to turbulence-inducing obstacles located immediately after the spark point, resulting in the emergence of a detonation wave from the obstacle section. This detonation wave 

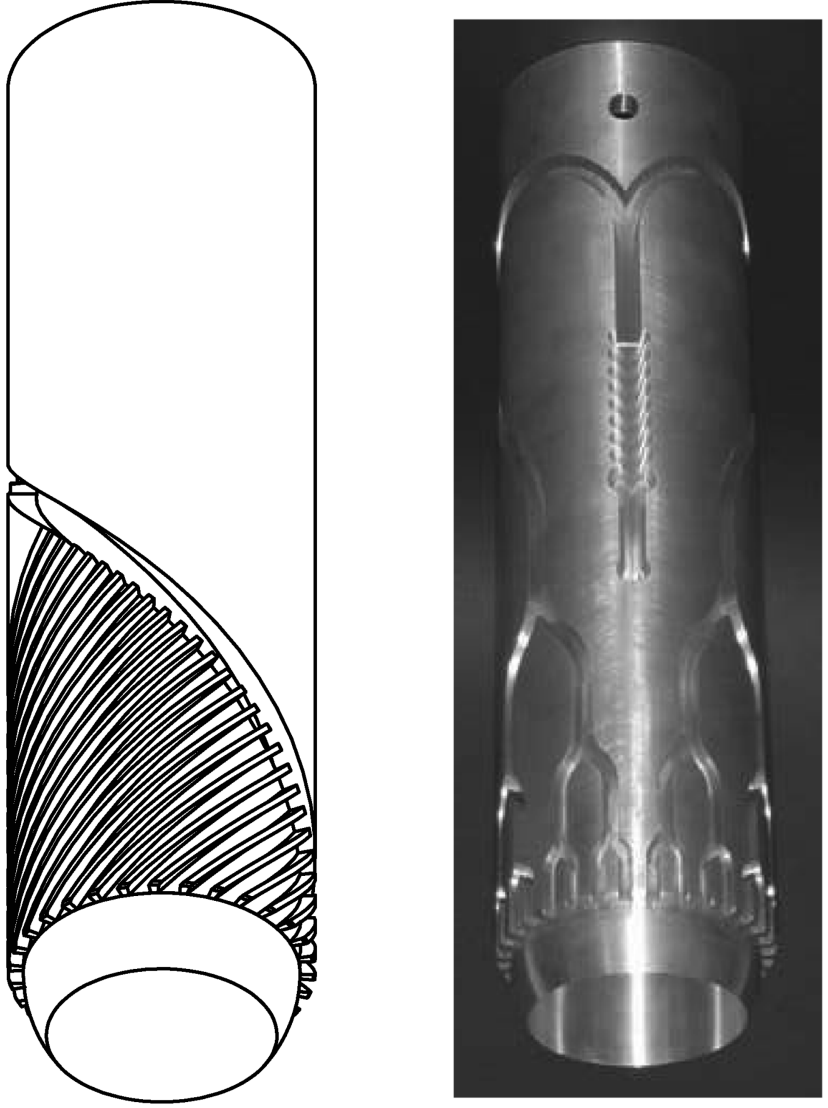

a)

b)

Fig. 2 The a) static toroidal initiator and the b) dynamic toroidal initiator.

then continued to travel throughout the device, branching off at each channel bifurcation. The distance between the spark point and the exit line (Fig. 3) was identical for each channel such that detonation wavelets from the initial source simultaneously passed the exhaust line and merged to form a ring-shaped detonation. A ramp located just past the exhaust line deflected this detonation ring inward toward its axis, sending an imploding detonation wave into the tube as shown in Fig. 4. Depending on the sensitivity of the tube mixture, this wave would either continue to propagate as an imploding detonation or shock wave.

\section{Testing Modes}

The toroidal initiator was tested with and without driver gas injection. Tests without gas injection were intended to characterize the imploding wave created in the initiator, while tests with gas injection evaluated the effectiveness of the imploding wave at detonating HC-air mixtures. Each of the test configurations is described below.

\section{Operation Without Gas Injection}

During testing without dynamic gas injection, the toroidal initiator was filled with stoichiometric propane-oxygen and ethylene-

Table 1 Channel dimensions of the dynamic initiator shown in Fig. 2b

\begin{tabular}{ccc}
\hline \hline Series no. & No. of channels & Channel width \\
\hline 1 & 1 & $10.2 \mathrm{~mm}$ \\
2 & 2 & $9.4 \mathrm{~mm}$ \\
3 & 4 & $8.4 \mathrm{~mm}$ \\
4 & 8 & $7.2 \mathrm{~mm}$ \\
5 & 16 & $6.1 \mathrm{~mm}$ \\
6 & 32 & $5.1 \mathrm{~mm}$ \\
\hline \hline
\end{tabular}

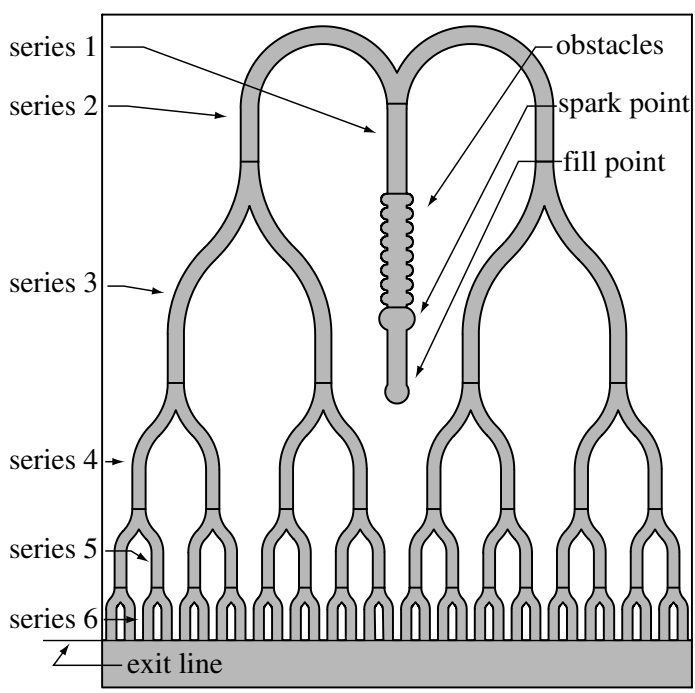

Fig. 3 The channel geometry of the dynamic initiator (Fig. 2b) mapped to a planar surface.

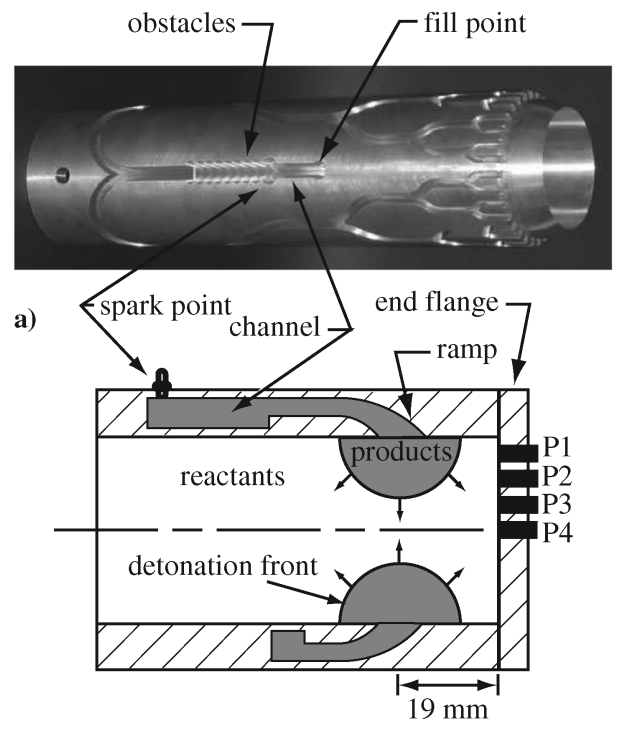

b)

Fig. 4 Toroidal initiator a) inner sleeve and b) schematic of the assembled device in operation. In the schematic, the gray areas are products, the white section is reactant, and the hatched areas are initiator walls. Pressure transducers are labeled P1, P2, P3, and P4.

oxygen mixtures to $0.10 \mathrm{MPa}$ initial pressure using the method of partial pressures. A bellows pump was used to recirculate the gas throughout the initiator to ensure homogeneity. After recirculation, the spark plug was discharged to ignite the mixture.

Pressure transducers and an intensified charge-coupled device (CCD) camera were used to observe the imploding wave. The end flange closest to the implosion could be instrumented with either pressure transducers or an optically clear window. When the experiment was set up to record pressure transducer measurements, the flange was outfitted with four pressure transducers (PCB 113A series) spaced along a radial line (Fig. 5). These pressure transducers were spaced $10.7 \mathrm{~mm}$ apart with the central transducer located at the flange center and were mounted on a surface that was $19 \mathrm{~mm}$ from the center of the exit of the initiator as shown in Fig. 4b. Pressure data were recorded with two Tektronics TDS 460 oscilloscopes at a sampling rate of $2.5 \mathrm{MHz}$. Labview software was used to drive the oscilloscopes and process the traces.

For optical access, the end flange containing the pressure transducers was replaced with a composite window consisting of a thin sacrificial layer and a thicker structural portion. The structural 


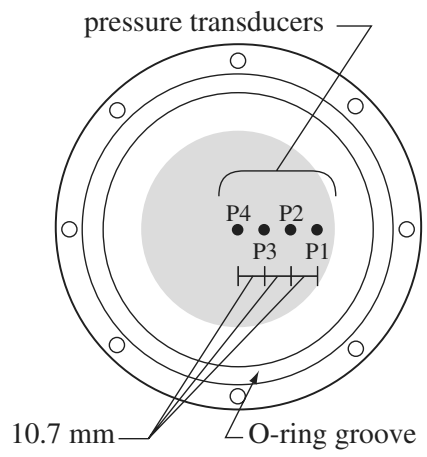

Fig. 5 A drawing of the end flange with the pressure transducer locations shown. The central transducer was a PCB model 113A24 and all other transducers were PCB model 113A26. The shaded region denotes the 76-mm-diam wetted area when the focusing ring is installed.

portion was a 31.8-mm-thick polycarbonate plate overlaid with a 6.35 -mm-thick aluminum ring to protect the polycarbonate from the stress concentrations induced by the fastening bolts. The sacrificial layer was a 6.35-mm-thick sheet of commercial glass sealed against the initiator via a 2.0-mm-thick Viton gasket. Previous testing [20] had determined that sacrificial glass windows were better able to withstand the heat generated by the combustion without charring compared to polycarbonate windows. After approximately a dozen tests, the glass window would develop cracks near the implosion focus, necessitating window replacement. For image acquisition, an intensified CCD (Princeton Instruments ITE/ICCD-576) camera was aligned with the centerline of the initiator and positioned a short distance outside the viewing window. The camera was triggered by a pressure transducer that was mounted at the exit line of the last series of channels.

\section{Operation with Gas Injection}

For tests with the gas injection, the initiator was attached to an extension tube to create a 1.0-m-long test-section tube, with $0.4 \mathrm{~m}$ made up by the toroidal initiator. The extension tube contained pressure transducers and ionization probes as shown in Fig. $\underline{6}$. Additional transducers were located on the flange near the implosion focus. The facility was filled with stoichiometric ethylene-oxygennitrogen and propane-oxygen-nitrogen test mixtures using the method of partial pressures and mixture homogeneity was accomplished by gas circulation via a bellows pump. After mixing, a gas injection system [29] injected an equimolar acetylene-oxygen
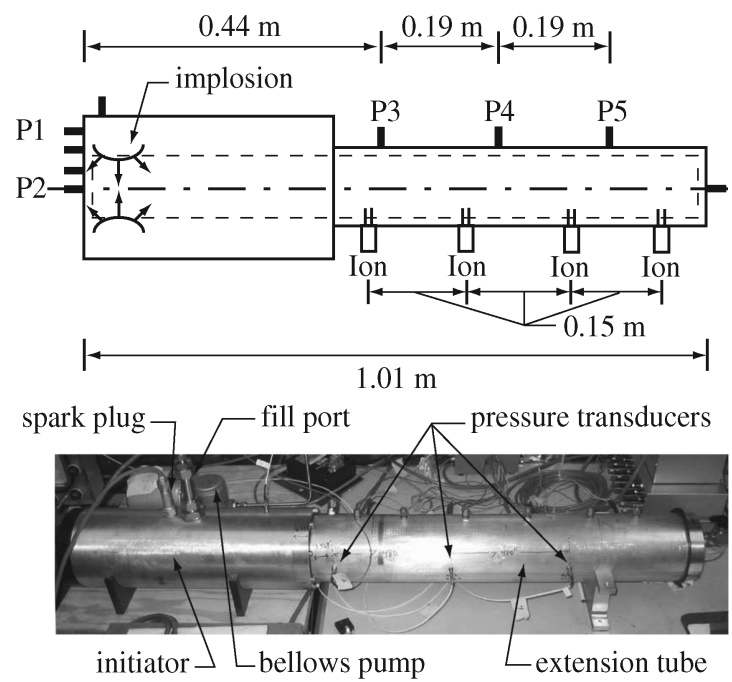

Fig. 6 A schematic and picture of the experimental setup used for initiation of $\mathrm{HC}$-air mixtures. The initiator is on the left; the extension tube is on the right. PCBs P3-P5 are spaced $19.0 \mathrm{~cm}$ apart. Ion probes are spaced $15.0 \mathrm{~cm}$ apart.

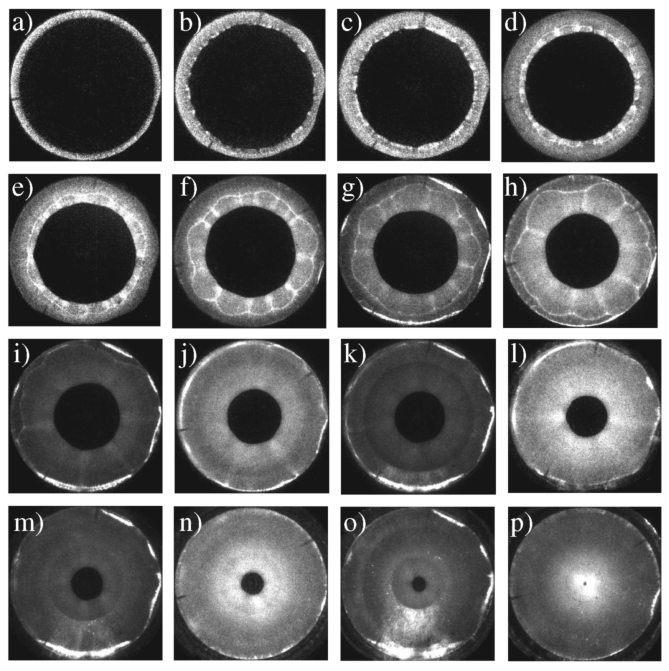

Fig. 7 Chemiluminescence images of a collapsing toroidal detonation wave in stoichiometric ethylene-oxygen mixtures at $0.10 \mathrm{MPa}$ initial pressure. Each image was acquired during a separate experiment and the period between the arrival of the detonation front at the triggering pressure transducer and imaging was a) $18 \mu \mathrm{s}$, b) $20 \mu \mathrm{s}$, c) $21 \mu \mathrm{s}$, d) $22 \mu \mathrm{s}$, e) $23 \mu \mathrm{s}$, f) $24 \mu \mathrm{s}$, g) $25 \mu \mathrm{s}$, h) $26 \mu \mathrm{s}$, i) $27 \mu \mathrm{s}$, j) $28 \mu \mathrm{s}$, k) $29 \mu \mathrm{s}$, 1) $30 \mu \mathrm{s}$, m) $31 \mu \mathrm{s}$, n) $32 \mu \mathrm{s}$, o) $33 \mu \mathrm{s}$, and p) $34 \mu \mathrm{s}$. Exposure times were $100 \mathrm{~ns}$.

mixture into the initiator channels for about $0.8 \mathrm{~s}$, displacing the test mixture. Immediately following gas injection, the spark plug was then discharged and pressure transducers and ionization probes located in the toroidal initiator and tube detected the resulting combustion front. Pressure and ionization data were recorded to a data acquisition system with a sampling rate of $1.0 \mathrm{MHz}$. No visualization was performed in this configuration.

\section{Results and Analysis}

\section{Toroidal Initiator Without Gas Injection}

Images obtained from testing with stoichiometric ethyleneoxygen mixtures without gas injection show a repeatable and regular collapsing circular front. A series of images of the imploding wave are shown in Fig. 7. The outermost black portion of each image is the initiator wall, which frames a 76-mm diam cross section of the tube. In each image, the innermost circle corresponds to the collapsing detonation front. In some images, a "flower-shaped" structure behind the collapsing front (between the innermost circle and the initiator wall) is also visible. This structure is attributed to detonation wave reflection from the window.

Although each image in Fig. 7 is from a separate experiment, multiple images of a single experiment were also recorded using a Cordin Model 220 gated, intensified camera that acquired an exposure every $2.5 \mu \mathrm{s}$ (Fig. 8). Those images were essentially identical to the single-run images (Fig. 7) and verified that with stoichiometric ethylene-oxygen mixtures at $0.10 \mathrm{MPa}$ initial pressure, the initiator operation is repeatable.

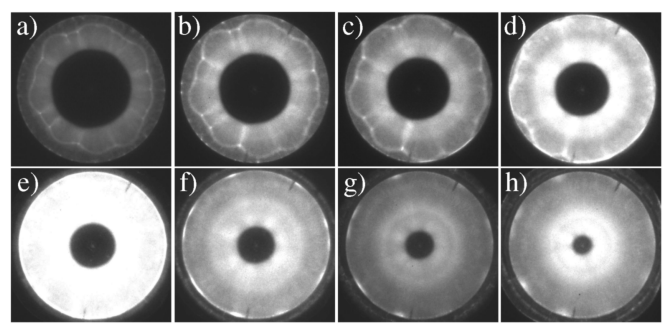

Fig. 8 Chemiluminescence images of a collapsing toroidal detonation wave in a stoichiometric ethylene-oxygen mixture at $0.10 \mathrm{MPa}$ initial pressure. Exposure times are $800 \mathrm{~ns}$ and all images were acquired during a single test. The period between the arrival of the detonation front at the triggering pressure transducer and imaging was a) $20.0 \mu \mathrm{s}$, b) $21.0 \mu \mathrm{s}$, c) $22.0 \mu \mathrm{s}$, d) $23.0 \mu \mathrm{s}$, e) $24.0 \mu \mathrm{s}$, f) $25.0 \mu \mathrm{s}$, g) $26.0 \mu \mathrm{s}$, and h) $27.0 \mu \mathrm{s}$. 


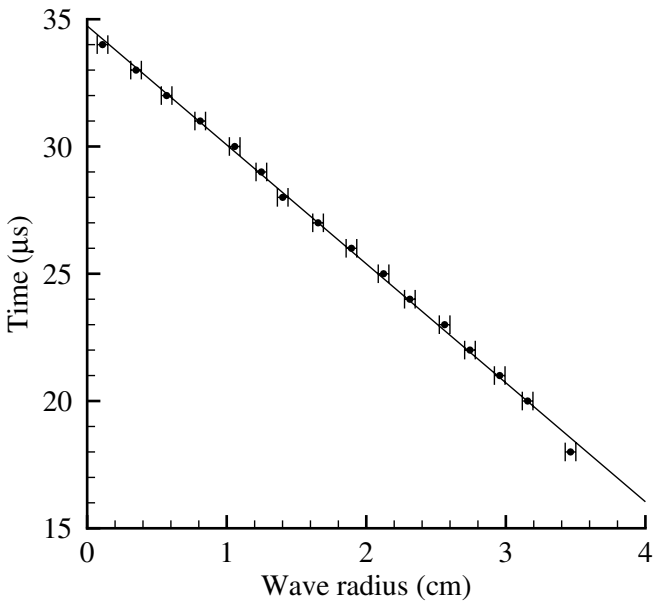

Fig. 9 A plot of wave radius as a function of time. Data are measured from the images shown in Fig. 7. The slope of the line fit to the data corresponds to a velocity of $2.20 \mathrm{~km} / \mathrm{s}$. The $C J$ wave speed $U_{\mathrm{CJ}}$ for the mixture is $2.40 \mathrm{~km} / \mathrm{s}$.

Measuring the wave radii shown in Fig. 7, it is possible to infer the wave speed of the collapsing front. Figure $\overline{9}$ contains a distance-time plot of the wave radius versus time. The data indicate that the wave is collapsing at a steady rate with a velocity of $2.20 \mathrm{~km} / \mathrm{s}$, which is $9 \%$ lower than the theoretical detonation wave speed of $2.40 \mathrm{~km} / \mathrm{s}$ predicted by CJ theory. Whitham's method is used below to explain this deficit, showing that, unlike cylindrical and spherical geometries, collapsing toroidal waves exhibit an initial period of velocity and pressure decay, later followed by a very short but intense period of overdrive at the end of the implosion process.

Although the initiator operation was highly repeatable with stoichiometric ethylene-oxygen mixtures, it was irregular with stoichiometric propane-oxygen mixtures at $0.10 \mathrm{MPa}$ initial pressure. Figure 10 shows a series of eight images taken by the Cordin model $22 \overline{0}$ camera during a single experiment where the initiator was filled with stoichiometric propane oxygen. In these experiments, the focus of the imploding wave was not aligned with the central axis of the initiator. Further investigation showed that the focal location of the imploding wave would wander from one experiment to another. When the "off-center" focus lined up with pressure transducers along the end flange, it was apparent that the device was still producing comparable focal pressures to tests where the focus was aligned in the center of the device.

Although the cause of the off-axis implosions was not definitively identified, it is likely that they were the result of the detonation wave weakening and slowing as it propagated through one of the $180 \mathrm{deg}$ turns in the initiator channels (shown on the left of Fig. 4a). In situations where the wave speed slowed more in one 180 deg turn than in the other, the wavelets would emerge from each half of the initiator channels at different times, creating the observed off-axis effect. This hypothesis is further supported by the observation that

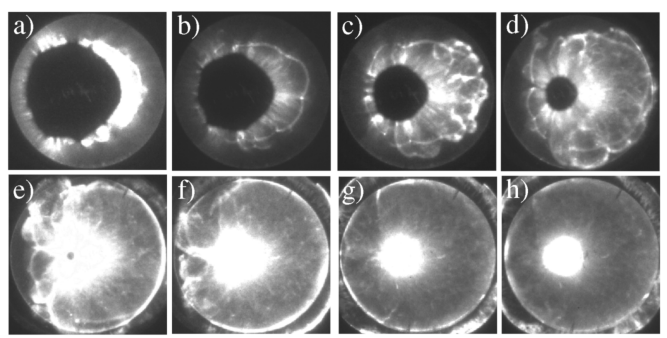

Fig. 10 Chemiluminescence images of collapsing toroidal detonation wave in a stoichiometric propane-oxygen mixture at 0.10 MPa initial pressure. Exposure times are $800 \mathrm{~ns}$ and all images were acquired during a single test. The period between the arrival of the detonation front at the triggering pressure transducer and imaging was a) $26.0 \mu \mathrm{s}$, b) $28.5 \mu \mathrm{s}$, c) $31.0 \mu \mathrm{s}$, d) $33.5 \mu \mathrm{s}$, e) $36.0 \mu \mathrm{s}$, f) $38.5 \mu \mathrm{s}$, g) $41.0 \mu \mathrm{s}$, and h) $43.5 \mu \mathrm{s}$.
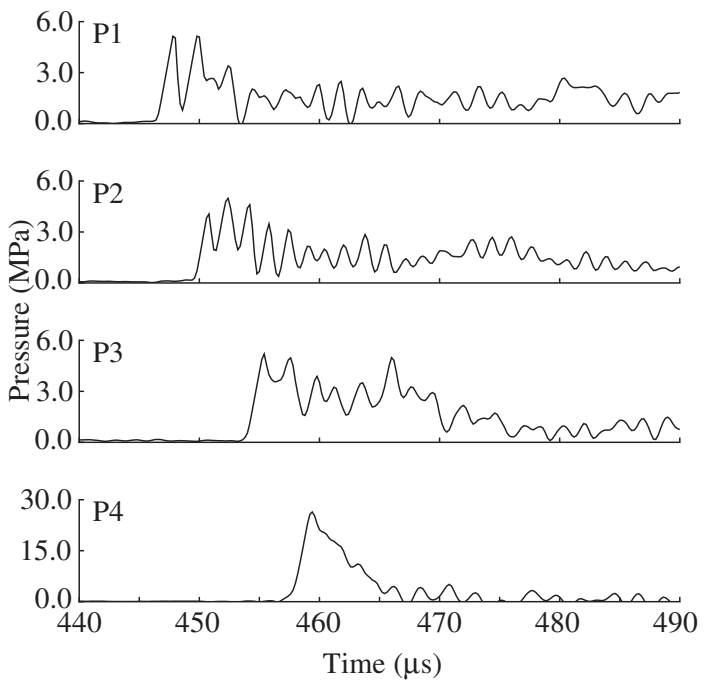

Fig. 11 Pressure traces from a typical initiator test with no gas injection. Both the initiator channels and the tube volume were filled with stoichiometric ethylene oxygen at 0.10 MPa. The CJ pressure for this mixture is 3.4 MPa. Pressure transducer locations are shown in Fig. 5.

during the collection of the imaging data, the initiator was aligned such that the wavelets coming from one $180 \mathrm{deg}$ turn would emerge on the left half of the image and the wavelets from the other $180 \mathrm{deg}$ turn would emerge from the right half of the image and all observed off-axis implosions were skewed to the left or right of the observation window. Additionally, the previously mentioned static initiator did not contain the $180 \mathrm{deg}$ turns and reliably produced regular implosions with propane-oxygen mixtures [20]. The $180 \mathrm{deg}$ turns only served to make the dynamic initiator more compact and are not essential to the design. Elevating the initial gas pressure would also increase the gas sensitivity and prevent wave failure through the 180 deg turn when testing with propane-oxygen mixtures.

Pressure measurements from the end flange transducers indicate that the implosion is producing a high-pressure focal region. A typical trace is shown in Fig. 11 from the toroidal initiator filled with a stoichiometric ethylene-oxygen mixture. Early in the implosion process, at $\mathrm{P} 1$, the peak wave pressure is $6.0 \mathrm{MPa}$, well above the $\mathrm{CJ}$ pressure $P_{\mathrm{CJ}}$ of $3.4 \mathrm{MPa}$. As the implosion process progresses, the pressure slightly decreases at $\mathrm{P} 2$ to about $4.5 \mathrm{MPa}$, before increasing to $30.0 \mathrm{MPa}$ (almost 10 times $P_{\mathrm{CJ}}$ ) at P4, the transducer closest to the focus. It is important to note that because the wave front is not normal to the pressure measurement plane, the pressure measurements record the interaction of the imploding wave with the end flange wall and do not directly measure the pressure behind the imploding wave along the implosion axis. The actual pressure evolution along the implosion axis can be deduced by consideration of the toroidal wave geometry during the implosion process, as is done in the next section.

\section{Theory and Simulations}

Although no theory and little analysis has been published on imploding toroidal waves [13], many studies have been done on cylindrical waves [14-18] due to the simplicity of the geometry. It is possible to imagine approximating the focal region of an imploding toroidal wave as an imploding cylindrical wave. In the following analysis, this assumption is compared to an approximate solution that is developed for an imploding toroidal wave. Experimental results from the toroidal initiator are also compared with the cylindrical and toroidal solutions and a numerical simulation is used to help explain the differences between the experimental data and idealized models.

Early research on imploding cylindrical waves focused on shock waves. In 1958, Whitham [11] developed a simple approximate solution to model the shock motion for a cylindrical imploding shock wave. His solution was based on an area-Mach number relationship for the wave and was derived by applying the equations of motion along a $C^{+}$characteristic behind the wave. The shock trajectory, pressure, and density were obtained using the shock-jump relations 
to show an inverse relationship between shock speed and shock area. Thus, as the area of the shock wave decreases, it becomes increasingly overdriven, generating elevated postshock pressures and flow velocities.

Whitham's work was extended to cylindrically imploding detonation waves by Lee and Lee [12]. Their model showed good agreement with their experiments, demonstrating that a collapsing cylindrical detonation wave is capable of producing pressures about 18 times higher than the normal CJ pressure.

For an imploding cylindrical detonation wave, Lee and Lee [12] reduced the Whitham model to

$$
\begin{aligned}
& \frac{1+\gamma \alpha+\gamma[(1+\gamma)(1-\alpha)]^{\frac{1}{2}}}{(1-\gamma \alpha)(1+\gamma)^{2}}\left[1+\gamma \alpha+\left(\frac{1+\gamma \alpha}{1-\alpha}\right)^{\frac{1}{2}}\right] d \alpha \\
& +\frac{d A}{A}=0
\end{aligned}
$$

where the variable $\alpha$ in Eq. (1) ranges from 0 to $1 / \gamma$, depending on the normalized wave speed $R_{s} / D$ and $\gamma$ :

$$
\alpha=\left[1-\left(D / \dot{R}_{s}\right)^{2}\right]^{\frac{1}{2}} / \gamma
$$

$D$ is the CJ detonation wave velocity,

$$
D=\left[2 Q\left(\gamma^{2}-1\right)\right]^{\frac{1}{2}}
$$

in the "strong-shock" limit.

It is necessary to solve Eq. (1) to determine the varying wave radius $R_{s}$. This can be done by relating the surface area of a collapsing cylindrical wave to the normalized shock radius $R_{s} / R_{i}$ where $R_{i}$ is the initial radius of the detonation wave:

$$
\frac{d A}{A}=\frac{d\left(R_{s} / R_{i}\right)}{\left(R_{s} / R_{i}\right)}
$$

Equation (4) is then substituted into the last term of Eq. (1), resulting in a differential equation in which the shock radius can be solved as a function of $\alpha$. The initial condition

$$
\alpha=0 \quad \text { at } \frac{R_{s}}{R_{i}}=1
$$

assumes that the wave starts as a $\mathrm{CJ}$ detonation.

Solving Eq. (1) for $\alpha$ as a function of $R_{s} / R_{i}$ with the boundary condition allows the density, particle velocity, and pressure behind the collapsing cylindrical detonation wave to be obtained as a function of $R_{s} / R_{i}$ from the shock-jump conditions:

$$
\begin{gathered}
\frac{\rho}{\rho_{i}}=\frac{1}{1-\alpha} \\
\frac{u}{u_{i}}=\frac{1+\gamma \alpha}{\left(1-\gamma^{2} \alpha^{2}\right)^{\frac{1}{2}}} \\
\frac{p}{p_{i}}=\frac{1}{1-\gamma \alpha}
\end{gathered}
$$

It is also possible to modify the solution of Lee and Lee [12] to approximate the motion of an imploding toroidal detonation wave. Equations (1-3) and (6-8) remain valid as they are independent of geometry. However, Eq. (4) needs to be adapted to the toroidal geometry.

For the geometry in Fig. 12, the differential area of a central element of the imploding toroidal detonation wave front initiated at a circle of radius $R_{i}$ can be represented as

$$
\Delta A=2 \pi R_{s} \cdot \Delta \theta\left(R_{i}-R_{s}\right)
$$

This is the equation for a parabola. Thus, as $R_{s}$ decreases (corresponding to an increase of the radius of the torus), the

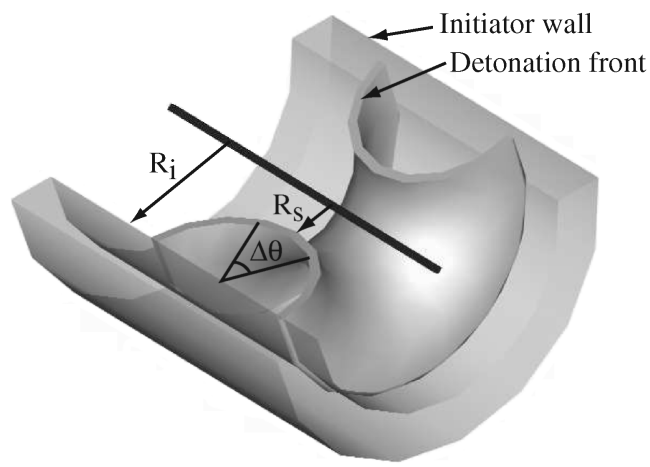

Fig. 12 The geometry of the toroidal detonation front.

differential surface area of a central element of the front first increases to a maximum at $R_{s}=R_{i} / 2$ and then decreases. With $A$ represented as a function of $R_{s}, d A / A$ can be represented in terms of $R_{s} / R_{i}$. However, directly solving for the flow as was done in the cylindrical case is not possible without an additional assumption that the detonation wave remains at $\mathrm{CJ}$ conditions throughout that region of increasing area $\left(R_{s} / R_{i}=0 \rightarrow 0.5\right)$. This is necessary because there are no solutions to the Whitham model [Eq. (1)] when $\dot{R}_{s}<D$.

Experimentally, a CJ detonation wave emerging from the annulus at $R_{i}$ could fail, becoming a nonreactive shock as its area increases. Although it is possible that the resulting decoupled shock and reaction zone could reinitiate a detonation following the region of area increase, such analysis is beyond the scope of this discussion. Instead, it is assumed that the detonation is ideal and remains at the CJ condition throughout the area-expansion process. Whitham's method is applied only to the wave from $R_{s} / R_{i}=0.5 \rightarrow 1$. In this regime, area is decreasing, resulting in an overdriven $\left(\dot{R}_{s}>D\right)$ detonation wave.

In practice, the likelihood of failure depends on the speed of the emerging detonation and the thickness of the detonation reaction zone as compared to the annular opening. Although no results are available in the literature for diffraction of waves through an annulus in the side wall of a tube, the results of Murray et al. [19] for an annular opening at the end of a tube should be a useful guide. If the detonation is propagating close to the $\mathrm{CJ}$ velocity, it will be transmitted as a detonation if the reaction zone length is sufficiently small compared to the width of the annular opening. If the reaction zone is too thick in comparison to the annular opening, failure of the detonation diffraction is anticipated. In the present case, the annular opening is about $12.7 \mathrm{~mm}$, which is 423 times larger than the estimated reaction zone length of $30 \mu \mathrm{m}$ for a stoichiometric propane-oxygen mixture at an initial pressure of 1 atm [30]. Based on previous diffraction experiments with slots [31], this should be adequate to achieve successful detonation. In terms of the more conventional approach of using the detonation cell width to characterize the opening, the cell width is about $0.9 \mathrm{~mm}$ and the opening is, therefore, about 14 cell widths, greater than the six to ten cell widths previously observed to be needed for successful diffraction from planar slots [31]. Furthermore, in the experimental part of this study, while the detonation wave was observed to propagate at a decreased velocity, it never failed during the implosion.

Experimental pressure data for the toroidal wave are plotted against the theoretical curves from Whitham's method for cylindrical and toroidal waves in Fig. 13. As exhibited in the experimental pressure history data of Fig. 11, the pressure of the toroidal detonation wave initially decays before increasing to almost 10 times $P_{\mathrm{CJ}}$ during the final stages of focusing. Neither the cylindrical theory nor the toroidal theory exhibit a pressure decay at any point during the focusing process, and the experimentally observed pressure decay is at least partly due to the location of the measurement plane off the central axis of the toroidal implosion, while the theoretical cylindrical and toroidal pressures were calculated on axis. Thus, the measured pressures show effects of off-axis diffraction and shock interaction with the end flange. The pressures measured by the 


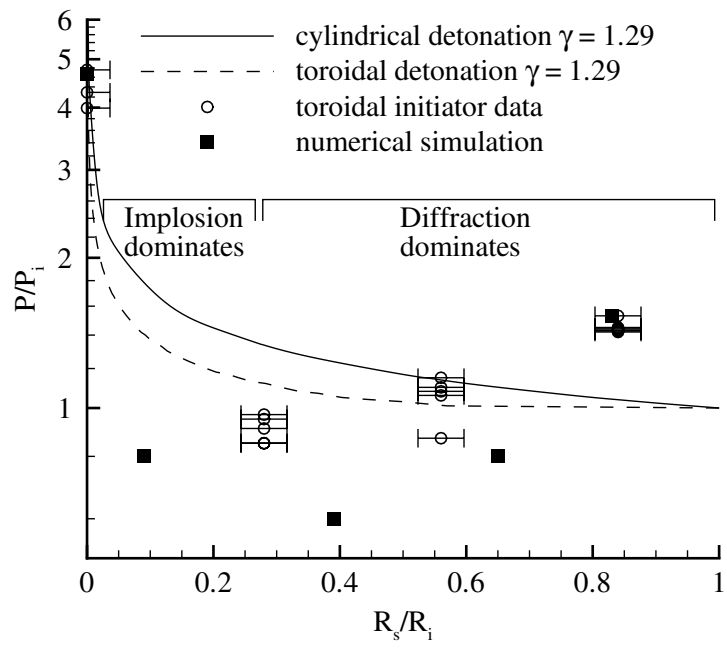

Fig. 13 Comparison of imploding cylindrical detonation theory, imploding toroidal detonation theory, toroidal initiator data with ethylene-oxygen mixtures and numerical simulations of an imploding shock.

transducers on the end flange can be significantly influenced by the angle the detonation wave makes with respect to the measurement surface. Only when the detonation front is normal to the wall can pressure be considered representative of the actual pressure in the undisturbed waves, as waves not perpendicular to the wall will form Mach stems or reflections.

A computer simulation of the experiment was used to help clarify this. A computationally simulated strong shock has been propagated through the geometry of the toroidal initiator with the computational fluid dynamics software AMRITA [32]. Although the simulation is for nonreacting flow, it demonstrates how shock interaction with the pressure-sensing wall can result in the observed pressures. A series of images of the simulated shock geometry and pressure profile along the right wall is shown in Fig. 14. The simulation assumes an ideal gas in two-dimensional, axisymmetric flow; the lower edge of each image is the axis of symmetry.

The simulation captures the experimental trend with initial pressure decay and the large increase in pressure toward the end of the focusing process. Figure 15 is a composite of several frames of the simulation showing the leading shock at four different times along with the locations of the pressure transducers in the experiment. Segments of the spatial pressure distribution are shown for each of these times. Note the interaction of the shock with the wall at each location.

Initially, near transducer P1, the shock wave exhibits almost complete normal reflection from the wall. Correspondingly, the measured pressure at location P1 is higher than the initial shock pressure. As the wave progresses, the reflection develops into a small Mach stem at location $P 2$, which results in a lower measured pressure than was recorded at P1. As the Mach stem increases in size, the measured pressure at the wall decreases. Between locations P3 and $\mathrm{P} 4$, the focusing processes, initially weak, begin to dominate the system, and the pressure rises dramatically. Previous work $[18,33,34]$ has shown that the reflection type will change from regular reflection to Mach reflection at the point where the included angle between the wave front and the wall is about $55 \mathrm{deg}$. For included angles between 0 and $55 \mathrm{deg}$, the peak pressure will be approximately $2.5 P_{\mathrm{CJ}}$. Between 55 and $90 \mathrm{deg}$, the pressure decreases monotonically to $P_{\mathrm{CJ}}$. Thus, this variation of pressure with wave angle is responsible for the peak pressure values greater than the CJ values as observed on the outer two transducers and the general trend from the four postshock pressure histories (Fig. 15) follows that of the postshock pressures measured in the toroidal initiator (Fig. 13).

The measured pressure evolution can be thought of as a combination of three processes: detonation-wall interaction, focusing effects, and diffraction. Initially, the wave diffracts and the detonation is not overdriven, and so focusing effects are weak.

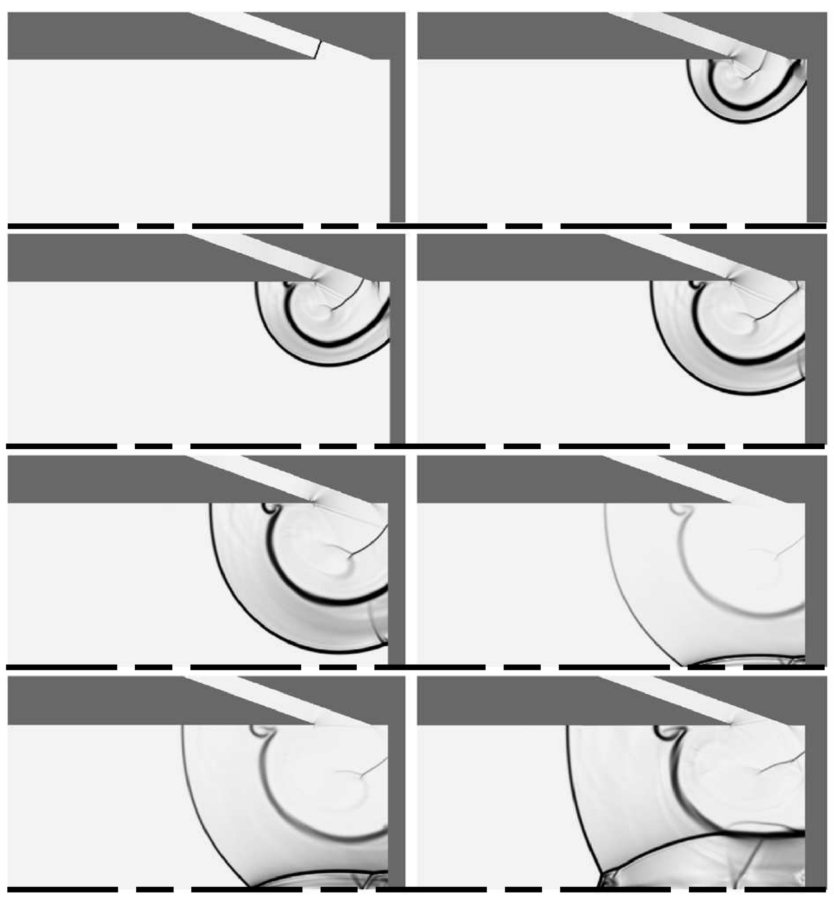

Fig. 14 A series of images from numerical simulations showing an imploding toroidal shock wave. The images are pseudo-schlieren visualizations of density gradients in the flow. The initial condition was a shock wave with $P_{2} / P_{1}=15$ and $T_{2} / T_{1}=10$.
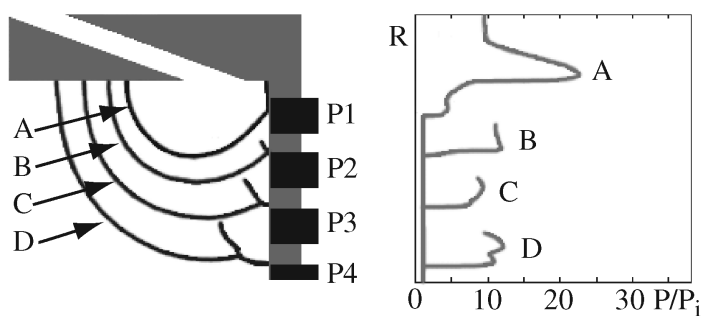

Fig. 15 This composite image shows the shock front at four different times. The corresponding spatial pressure profiles are also plotted. Transducers are not to scale.

Detonation-wall interactions dominate the measured pressure, leading to the apparent pressure decay. Later on in the process, after the Mach stem has developed, focusing processes significantly overdrive the wave and dramatically increase the pressure. It is important to note that the apparent pressure decay due to the wall reflection is not actually present along the focal axis of the device, whereas the pressure increase due to focusing is present. Also, the experimental data points show that the detonation wave experiences significantly less diffraction than the shock wave in the simulation, which supports the assumption that the reaction front remains coupled and continues to support the toroidal detonation wave throughout the period of wave expansion.

\section{Toroidal Initiator with Gas Injection}

During testing, the amount of diluent in the tube mixture and the amount of injected acetylene-oxygen gas were varied. The main criterion for successful initiation of the test-section mixture was that the experimentally measured wave speed be not more than $10 \%$ below the $\mathrm{CJ}$ detonation velocity $U_{\mathrm{CJ}}$ for the tube mixture. The experimental uncertainty in the velocity measurements was less than $6 \%$. If this criterion was met, the peak pressure of the wave was examined to ensure that it was on the order of $P_{\mathrm{CJ}}$ for the test-section mixture. Additionally, ionization probe traces were used to verify that the shock wave measured by the pressure transducers was accompanied by a tightly coupled reaction zone. The measured wave 


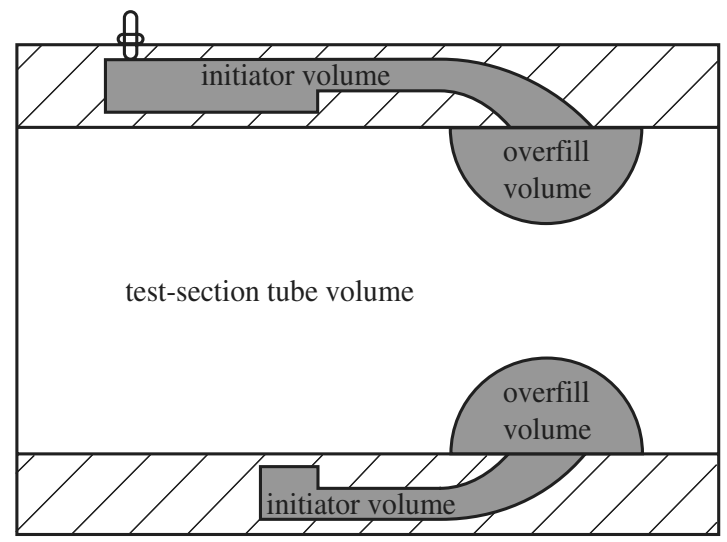

Fig. 16 The overfilled initiator gas shown as semicircular volumes in the test-section tube.

speed used in the above criterion was found by averaging the wave speeds measured between P3 and P4 and between P4 and P5. (Transducer locations are shown in Fig. 6.)

The amount of acetylene-oxygen initiator gas used in each experiment is presented in terms of "initiator overfill." This refers to the amount of gas injected into the experiment that was in excess of the initiator volume. A graphical interpretation of this concept is illustrated in Fig. 16. Negative values of initiator overfill correspond to the initiator channels not being completely filled with initiator gas. The effective volume of the initiator channels is $354 \mathrm{cc}\left(21.6\right.$ in. $\left.^{3}\right)$, which accounts for the actual volume of the initiator $(218 \mathrm{cc}$ or 13.3 in. $^{3}$ ) and the volume of the tubing associated with the gas injection system ( $136 \mathrm{cc}$ or $8.3 \mathrm{in}^{3}{ }^{3}$ ). The total system volume of the initiator and the tube is $4980 \mathrm{cc}\left(304 \mathrm{in}^{3}\right)$. An example of the overfill volume calculation is provided for clarity: An overfill volume of $37 \%$ corresponds to the effective initiator volume in addition to $37 \%$ of the actual initiator volume:

$$
354 \mathrm{cc}+0.37(218 \mathrm{cc})=435 \mathrm{cc}
$$

Thus, immediately after injection, the initiator is completely filled with driver gas and an additional $81 \mathrm{cc}$ of this acetylene-oxygen gas has spilled into the tube volume.

\section{Example Data Traces}

The result of each run was classified as either a successful initiation or a failed initiation depending on whether a detonation was detected in the tube. To better characterize the wave created in the tube by the initiator in the absence of combustion, calibration runs were also conducted with the tube filled with only nitrogen.

Figure 17 shows several pressure transducer and ionization probe traces from such a calibration run. Each trace is labeled and corresponds to a transducer shown in Fig. 6. All ionization probe data are on the same data acquisition channel. It is assumed that the ionization probes were triggered sequentially from left to right as they are shown in Fig. 6. Pressure transducers P1 and P2 show data characteristic of the imploding wave and measure pressures on the order of 10.0 MPa near the focal region. The implosion generates a shock wave in the tube that decays as it propagates down the length of the tube. The flow behind the shock wave has an overpressure of $0.4 \mathrm{MPa}$ that agrees with the $0.65 \mathrm{~km} / \mathrm{s}$ measured shock velocity corresponding to a Mach 2 shock wave. The ionization probes indicate no ionization is present, as would be expected from an inert mixture processed by a weak shock wave.

Figure 18 shows data from a run with propane air in the tube where a detonation was not successfully transmitted from the initiator to the test-section mixture. Initiator overfill in this experiment was $24 \%$ of the initiator volume. The data configuration is the same as with the previous example. Pressure transducer P1 shows a typical detonation wave that is overdriven to a very high pressure $(20.0 \mathrm{MPa})$ as it implodes near pressure transducer P2. Farther down the tube, a shock wave with an overpressure of $0.7 \mathrm{MPa}$ is present. As the wave
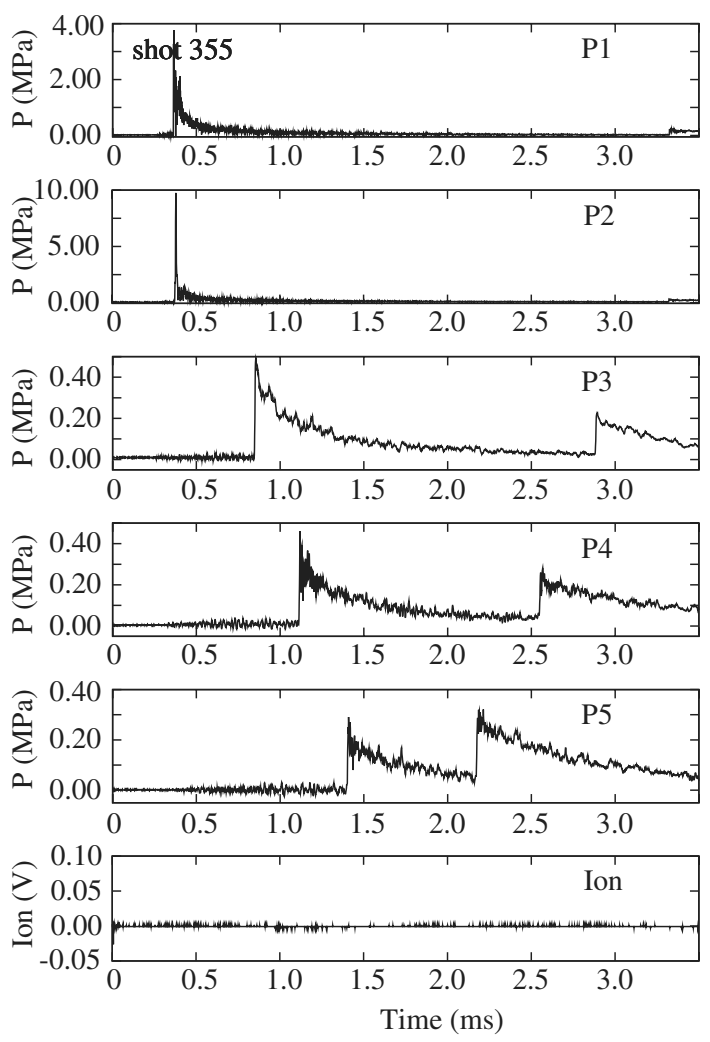

Fig. 17 Pressure and ionization traces from a typical calibration shot. Test-section mixture was nitrogen at $0.10 \mathrm{MPa}$ initial pressure. Traces are labeled and correspond to locations shown in Fig. $\underline{6}$.
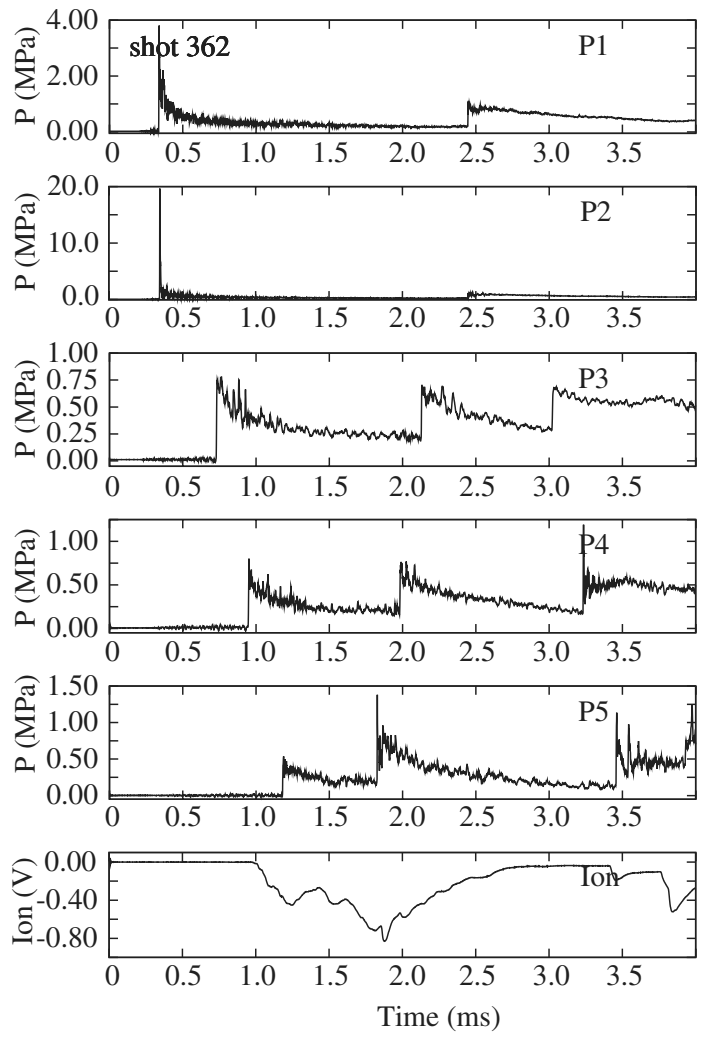

Fig. 18 Pressure and ionization traces from a typical failed initiation. Test-section mixture was stoichiometric propane air at 0.10 MPa initial pressure. Traces are labeled and correspond to locations shown in Fig. $\underline{6}$. 

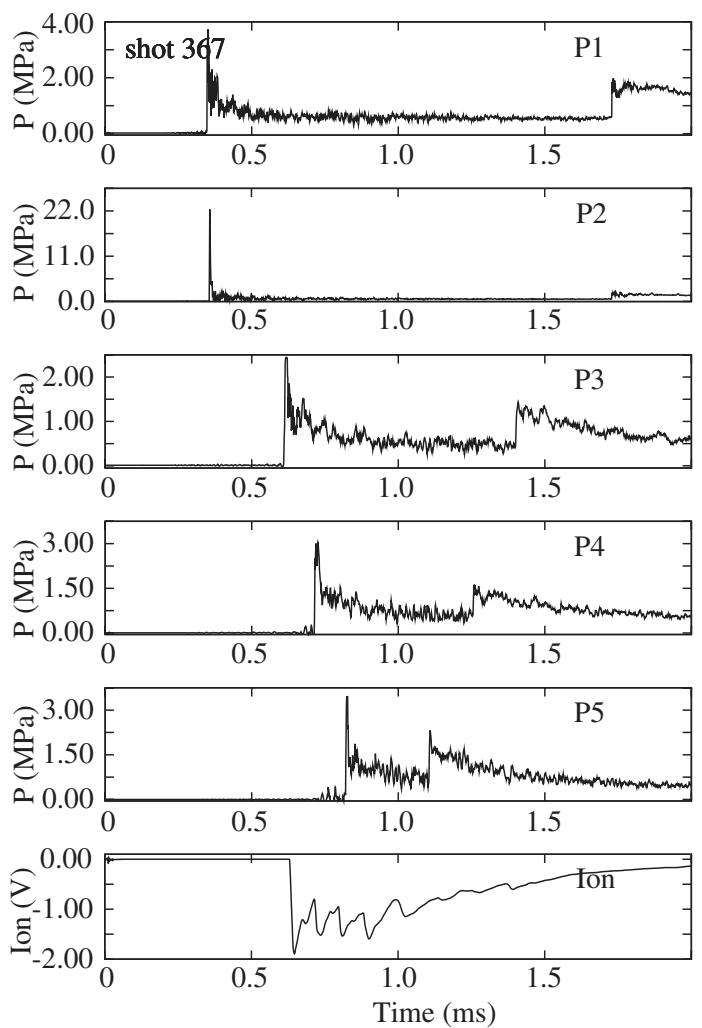

Fig. 19 Pressure and ionization traces from a typical successful initiation. Test-section mixture was stoichiometric propane air at 0.10 MPa initial pressure. Traces are labeled and correspond to locations shown in Fig. 6.

propagates the length of the tube, it decays. The $0.7 \mathrm{MPa}$ overpressure is higher than the $0.4 \mathrm{MPa}$ overpressure measured in the calibration case where no combustion was present; however, it is far below the CJ pressure of 1.9 MPa for stoichiometric propane-air mixtures. Inspection of the ionization probe data shows the broad dips characteristic of a deflagration. Furthermore, the measured wave speeds are on the order of $0.80-1.00 \mathrm{~km} / \mathrm{s}$, while CJ theory predicts $U_{\mathrm{CJ}}$ to be $1.80 \mathrm{~km} / \mathrm{s}$. Thus, in this experiment, a detonation did not propagate down the length of the tube. Instead, a shock wave was present, followed by a deflagration.

Data from an experiment where a propane-air test-section mixture was successfully detonated are shown in Fig. 19. The initiator overfill in the experiment was $37 \%$ of the volume of the initiator. The data configuration is the same as in previous examples. Pressure transducers located near the implosion focus register the same highpressure focal region as in previous cases. This time, however, pressure transducer $\mathrm{P} 3$ records the passing of a wave with an overpressure of $2.5 \mathrm{MPa}$ which is $30 \%$ above $P_{\mathrm{CJ}}$. This wave maintains its overpressure as it continues to propagate down the length of the tube. Measured wave speeds of $1.81 \mathrm{~km} / \mathrm{s}$ agree well with $U_{\mathrm{CI}}(1.80 \mathrm{~km} / \mathrm{s})$. Furthermore, the ionization probe traces show the sharp spike characteristic of a detonation wave and also indicate that the combustion front is coupled with the pressure wave.

\section{Transmission Limits}

During investigation of the transmission efficiency of the initiator, the amount of initiator gas injected into the device and the wall proximity to the implosion focus were varied. To vary the wall proximity to the focus, two experimental configurations were used. In the first, the focus was only $19 \mathrm{~mm}$ from the end flange (Fig. 20a). It was thought that the end flange would enhance the focusing by providing an additional surface to reflect the waves. To remove this effect, separate tests were conducted with the initiator flipped around such that the focus was about $0.4 \mathrm{~m}$ from the end flange (Fig. 20b). In experiments with the focus at the end flange, stoichiometric propane-oxygen and ethylene-oxygen mixtures were used with

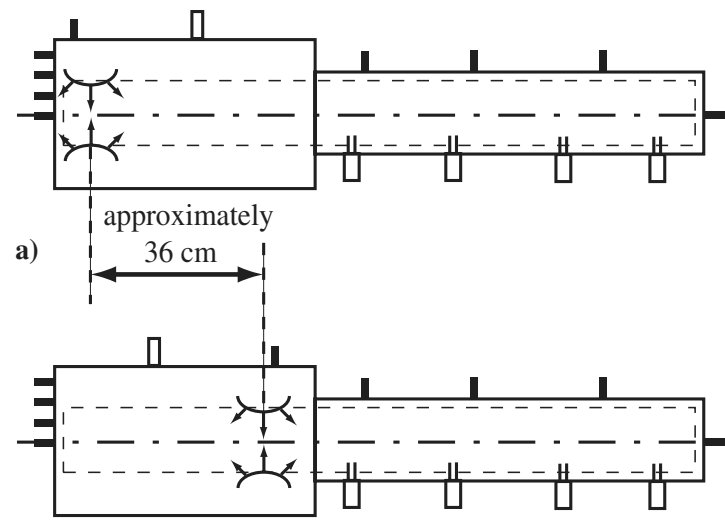

b)

Fig. 20 Schematics illustrating the difference in the focal location of the imploding wave when the focus was a) near the end wall and b) far from the end wall.

varying amounts of nitrogen dilution. Experiments with the focus far from the wall tested only stoichiometric propane-air and ethyleneair mixtures. The results are separated into four categories according to wall proximity (wall focusing or no wall focusing) and fuel used in the tube (propane or ethylene).

1) Propane mixtures with focus near the wall: Experimental results for the wave focus next to the end flange wall with propane mixtures are shown in Table 2 . The average wave velocity in the tube is compared to the amount of diluent present in the test-section mixture (by mol percent). The table clearly shows that as the amount of diluent is increased, it is necessary to inject more initiator gas to achieve a stronger initiation event. The minimum amount of initiator gas that was able to initiate stoichiometric propane air was found to correspond to an initiator overfill of $37 \%$. Experiments with propane air are presented on a separate plot (Fig. 21) to more clearly visualize the threshold. The wave appears to be overdriven near the initiation threshold, hinting at the presence of a galloping wave, a phenomenon that occurs in marginal detonations. Unfortunately, velocity measurements did not have sufficient resolution, and the tube was not of sufficient length to study this effect in detail. Assuming the critical amount of gas overfill was confined to a cylindrical slug at $0.1 \mathrm{MPa}$ with the same diameter as the inside of the tube $(76 \mathrm{~mm})$, the width of the cylinder would be $2.8 \mathrm{~cm}$ (1.1 in.).

2) Ethylene mixtures with focus near the wall: Results with ethylene fuel (Table 3 ) followed the same trend as the propane cases; however, due to the increased sensitivity of ethylene-oxygen mixtures, less initiator gas was required to initiate stoichiometric ethylene-air mixtures. The critical overfill value was determined to

Table 2 Wave speed in the test-section tube as a function of test gas diluent and initiator overfill for stoichiometric propane-oxygennitrogen mixtures with the focus near the end wall. The second row denotes percent moles of nitrogen in the test-section tube mixture. The first column denotes initiator overfill. Values of $U_{\mathrm{CJ}}$ for each dilution are listed on the bottom row. All other values are wave speeds (in $\mathrm{km} / \mathrm{s}$ ) measured in the test-section tube. Wave speeds within $10 \%$ of $U_{\mathrm{CJ}}$ are considered detonations. If a cell is filled with "X," no experiment was performed at that condition

\begin{tabular}{ccccc}
\hline \hline & \multicolumn{4}{c}{ Diluent (mol \%) } \\
Initiator overfill & $50 \%$ & $60 \%$ & $70 \%$ & $75.8 \%$ \\
\hline $6 \%$ & 2.08 & 0.94 & 0.81 & $\mathrm{X}$ \\
$15 \%$ & $\mathrm{X}$ & 2.00 & 0.85 & $\mathrm{X}$ \\
$24 \%$ & $\mathrm{X}$ & $\mathrm{X}$ & 1.89 & 0.84 \\
$32 \%$ & $\mathrm{X}$ & $\mathrm{X}$ & $\mathrm{X}$ & 0.85 \\
$37 \%$ & $\mathrm{X}$ & $\mathrm{X}$ & $\mathrm{X}$ & 2.16 \\
$41 \%$ & $\mathrm{X}$ & $\mathrm{X}$ & $\mathrm{X}$ & 1.81 \\
$49 \%$ & $\mathrm{X}$ & $\mathrm{X}$ & $\mathrm{X}$ & 1.78 \\
$61 \%$ & $\mathrm{X}$ & $\mathrm{X}$ & $\mathrm{X}$ & 1.78 \\
$73 \%$ & $\mathrm{X}$ & $\mathrm{X}$ & $\mathrm{X}$ & 1.82 \\
$U_{\mathrm{CJ}}$ & 2.06 & 1.98 & 1.88 & 1.80 \\
\hline \hline
\end{tabular}




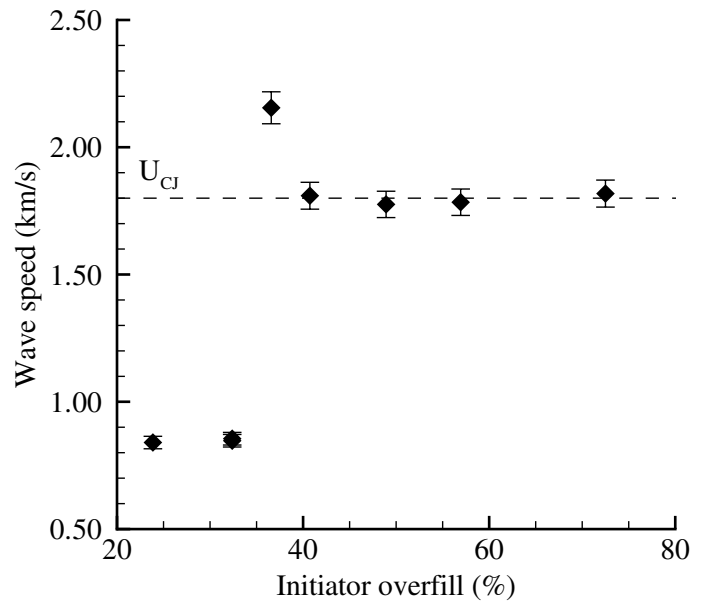

Fig. 21 Wave speed in the tube as a function of initiator overfill for stoichiometric propane-air mixtures with the focus near the end wall. $U_{\mathrm{CJ}}$ is $1.80 \mathrm{~km} / \mathrm{s}$.

be $6 \%$. Fuel-air cases are plotted alone in Fig. 22. No overdriven waves are present in this case. The critical amount of overfill corresponds to a disk of diameter $76 \mathrm{~mm}$ and width $0.45 \mathrm{~cm}(0.18 \mathrm{in})$.

3) Propane mixtures with focus far from the wall: Increasing the distance of the end flange wall from the focusing event necessitated more initiator gas being injected to detonate propane-air mixtures. Figure 23 shows steadily increasing test-section wave velocities as initiator overfill is increased. The critical amount of overfill required for initiation of the propane-air mixture was found to be $73 \%$. It should be noted that while this is almost twice the critical overfill percent value for cases with the focus located next to the wall, twice the amount of gas was not injected. Instead, it means that only twice the amount of overfill gas was injected. The critical amount of overfill for this case corresponds to a cylinder of diameter $76 \mathrm{~mm}$ and a width of $5.5 \mathrm{~cm}(2.2 \mathrm{in}$.).

4) Ethylene mixtures with wave focus far from the wall: As with the propane cases, distancing the end flange wall from the wave focus required more gas to be injected to initiate the ethylene-air mixture in the tube (Fig. 24). The critical amount of overfill was found to be $20 \%$, corresponding to a cylinder with a diameter of $76 \mathrm{~mm}$ and a width of $1.5 \mathrm{~cm}(0.59$ in.). Table 4 summarizes the above results, comparing the amount of overfill necessary for detonation transmission from the initiator to the tube with and without wall focusing for different fuels. Table 5 contains the length of the tube that the total amount of gas used in the entire initiation process would fill, were it injected directly into the test-section tube (Fig. 25), as would be done with a simple tube initiator.

Table 3 Wave speed in the test-section tube as a function of test gas diluent and initiator overfill for stoichiometric ethylene-oxygennitrogen mixtures with the focus near the end wall. The second row denotes percent moles of nitrogen in the test-section tube mixture. The first column denotes initiator overfill. Values of $U_{\mathrm{CJ}}$ for each dilution are listed on the bottom row. All other values are wave speeds (in $\mathbf{~ m m} / \mathbf{s}$ ) measured in the test-section tube. Wave speeds within $10 \%$ of $U_{\mathrm{CJ}}$ are considered detonations. If a cell is filled with $X$, no experiment was performed at that condition

\begin{tabular}{ccccc}
\hline \hline & \multicolumn{4}{c}{ Diluent $(\mathrm{mol} \%)$} \\
Initiator overfill & $50 \%$ & $60 \%$ & $70 \%$ & $73.8 \%$ \\
\hline$-30 \%$ & 2.05 & 1.98 & 0.63 & 0.59 \\
$-7 \%$ & $\mathrm{X}$ & $\mathrm{X}$ & $\mathrm{X}$ & 0.83 \\
$3 \%$ & $\mathrm{X}$ & $\mathrm{X}$ & $\mathrm{X}$ & 0.85 \\
$5 \%$ & $\mathrm{X}$ & $\mathrm{X}$ & $\mathrm{X}$ & 0.86 \\
$6 \%$ & $\mathrm{X}$ & $\mathrm{X}$ & $\mathrm{X}$ & 1.84 \\
$15 \%$ & $\mathrm{X}$ & $\mathrm{X}$ & $\mathrm{X}$ & 1.85 \\
$61 \%$ & $\mathrm{X}$ & $\mathrm{X}$ & $\mathrm{X}$ & 1.79 \\
$U_{\mathrm{CJ}}$ & 2.06 & 1.98 & 1.87 & 1.82 \\
\hline \hline
\end{tabular}

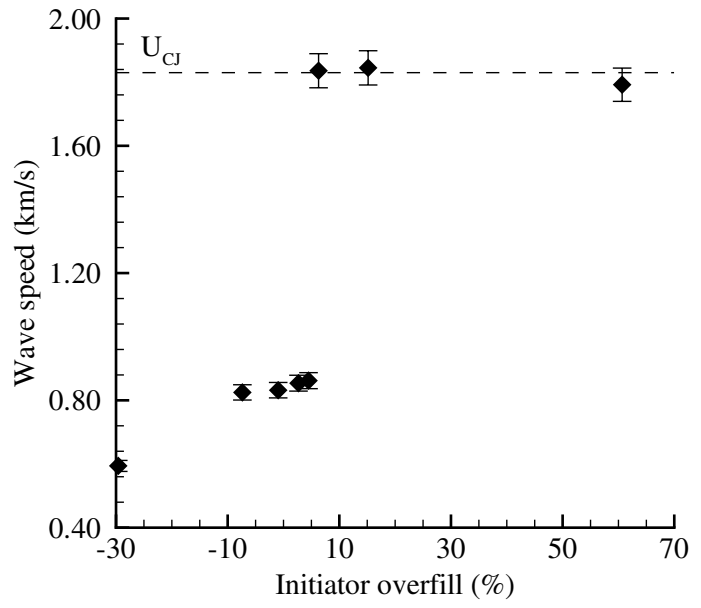

Fig. 22 Wave speed in the tube as a function of initiator overfill for stoichiometric ethylene-air mixtures with the focus near the end wall. $U_{\mathrm{CJ}}$ is $1.83 \mathrm{~km} / \mathrm{s}$.

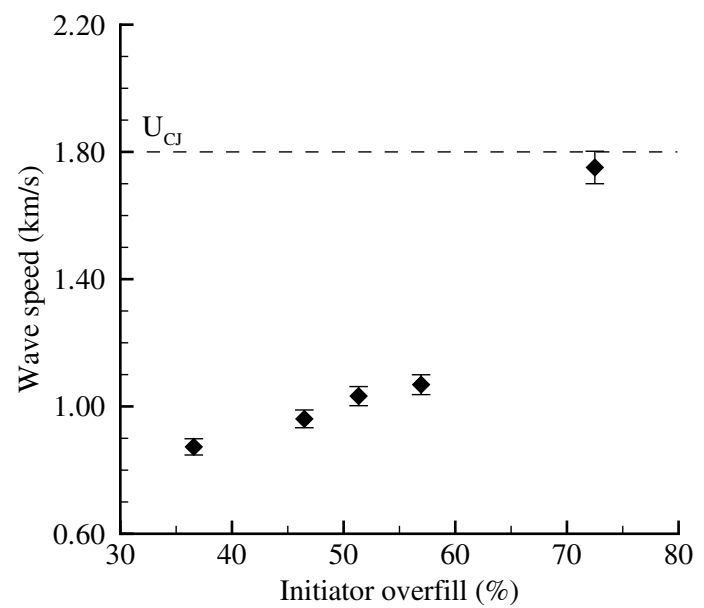

Fig. 23 Wave speed in the tube as a function of initiator overfill for stoichiometric propane-air mixtures with the focus far from the wall. $U_{\mathrm{CJ}}$ is $1.80 \mathrm{~km} / \mathrm{s}$.

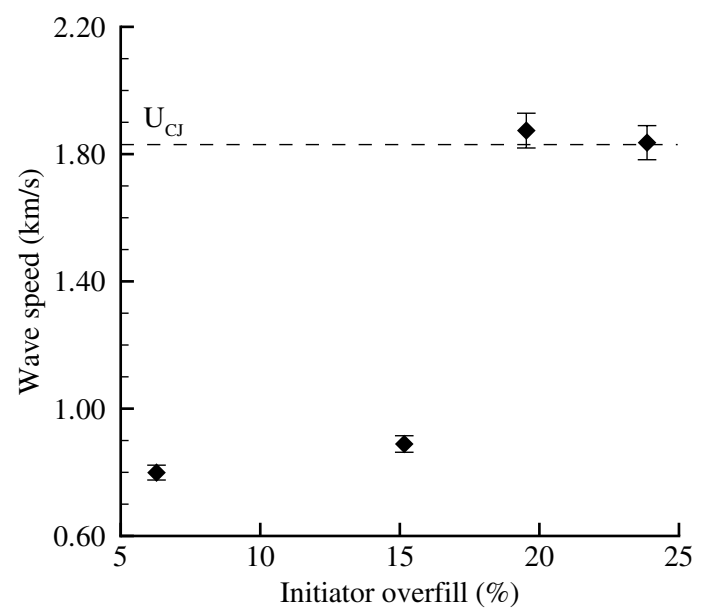

Fig. 24 Wave speed in the tube as a function of initiator overfill for stoichiometric ethylene-air mixtures with the focus far from the wall. $U_{\mathrm{CJ}}$ is $1.83 \mathrm{~km} / \mathrm{s}$.

The proximity of the end flange wall is thought to reduce the amount of gas required for initiation by creating symmetry and by promoting wave reflection. In a situation of perfect symmetry (Fig. 26), the wave focus would occur at the end flange wall and the overfill volume would be half of the case where no end wall was 
Table 4 Critical amount of overfill necessary for detonation initiation with different experimental configurations

\begin{tabular}{lcc}
\hline & Near wall & Far from wall \\
\hline $\mathrm{C}_{3} \mathrm{H}_{8}$-air & $37 \%$ & $73 \%$ \\
$\mathrm{C}_{2} \mathrm{H}_{4}$-air & $6 \%$ & $20 \%$ \\
\hline \hline
\end{tabular}

Table 5 Length of $76 \mathrm{~mm}$ tube that would be filled by a critical amount of initiator gas were the gas injected directly into the tube as shown in Fig. 25

\begin{tabular}{lcc}
\hline \hline & Near wall & Far from wall \\
\hline $\mathrm{C}_{3} \mathrm{H}_{8}$-air & $9.3 \mathrm{~cm}$ & $11.0 \mathrm{~cm}$ \\
$\mathrm{C}_{2} \mathrm{H}_{4}$-air & $7.8 \mathrm{~cm}$ & $8.5 \mathrm{~cm}$ \\
\hline \hline
\end{tabular}

present. However, in the actual experiments with the wave focus near the end wall, the wave focus was still $19 \mathrm{~mm}$ away from the end wall, and only partial symmetry was achieved.

In addition to symmetry, the proximity of the end wall to the focus also enhances the initiation by providing an additional surface from which the exploding waves can reflect. This additional level of confinement further reduces the overfill volume. From Table 4 , it can be seen that for propane-air mixtures, these two effects reduced the overfill volume by half when the focus was near the wall. For ethylene-air mixtures, the overfill reduction is even more dramatic; initiation near the wall requires only one-third the overfill volume compared to situations where the focus was far from the end wall.

\section{Initiation Attempts Using a Collapsing Shock Wave}

The initiator was also used to generate an imploding shock wave in an attempt to initiate the test-section mixture. Recent computational

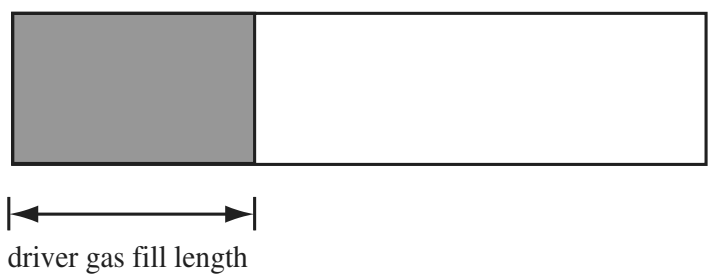

Fig. 25 Accompanying schematic for Table 5, where a critical amount of initiator gas (gray) is injected into the test-section tube directly.
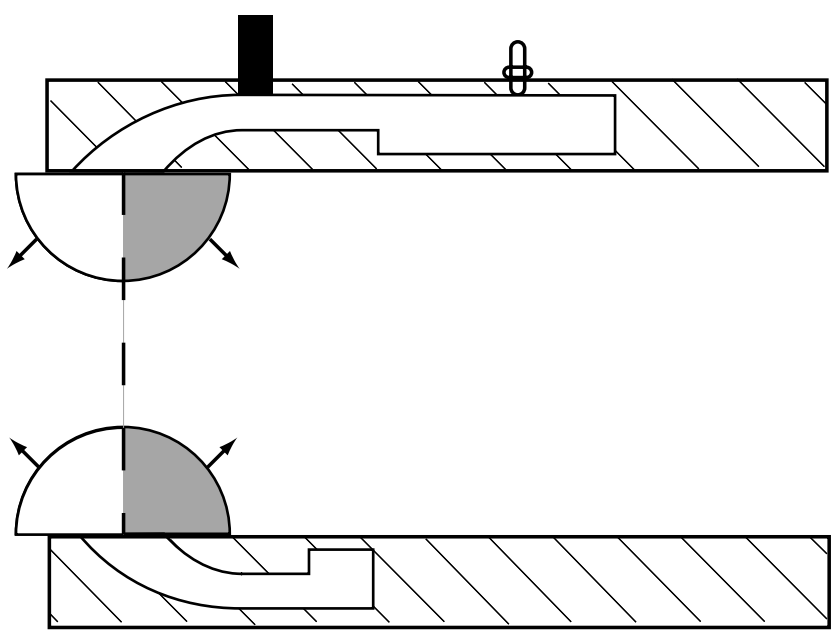

Fig. 26 A schematic demonstrating the reduction in overfill volume due to symmetry. For pure symmetry, the end flange wall would be located at the dashed line. The volume of overfill gas would be reduced to the gas shaded gray, which is half of the volume required in the case where no end flange wall is present. simulations by $\mathrm{Li}$ and Kailasanath [35] have suggested that it is possible to initiate JP10-air mixtures using impulsively started jets of JP10 and air to create an annular shock wave.

While this concept was experimentally studied in detail in later work [23], a preliminary investigation of this notion was examined by conducting imploding shock experiments with the present setup. To generate an imploding shock wave, the initiator was partially filled (roughly $30-40 \%$ ) with initiator gas. Detonation of this gas propagated a shock wave followed by a deflagration through the channels of the device. This shock wave then imploded at the focus, creating an imploding shock wave in the fuel-air mixture.

This technique was unsuccessful at initiating stoichiometric ethylene-air mixtures. Pressure traces from an experiment where $41 \%$ of the initiator was filled with initiator mixture are shown in Fig. 27. The location of the pressure and ionization probe traces from Fig. $\overline{27}$ are shown in the schematic in Fig. 28 . The tube mixture was ethylene air.

Pressure transducer $\mathrm{P} 2$ shows a shock wave with an overpressure of 1.2 MPa that is propagated into the test-section mixture from the initiator. As this wave implodes, the pressure measured near the focus is $10.0 \mathrm{MPa}$. Farther down the tube, pressure transducers P4 and P5 show a shock with an overpressure of $0.4 \mathrm{MPa}$. Measured wave speed in the tube is roughly $0.63 \mathrm{~km} / \mathrm{s}$ while $U_{\mathrm{CJ}}$ is $1.83 \mathrm{~km} / \mathrm{s}$. Thus, initiation of the tube mixture was not successful as the pressure traces are similar to those previously presented for the "failed initiation" case, where an imploding detonation wave (instead of a shock wave) was propagated into the tube. In these experiments, the imploding shock wave was not of sufficient Mach number and the postshock flow was not of sufficient duration to initiate the ethyleneair mixture. This concept is addressed more carefully using a different experimental geometry in other work [23].

\section{Experimental Uncertainty}

The dominant source of experimental uncertainty was due to the sampling rate and finite size of the pressure transducers used to detect
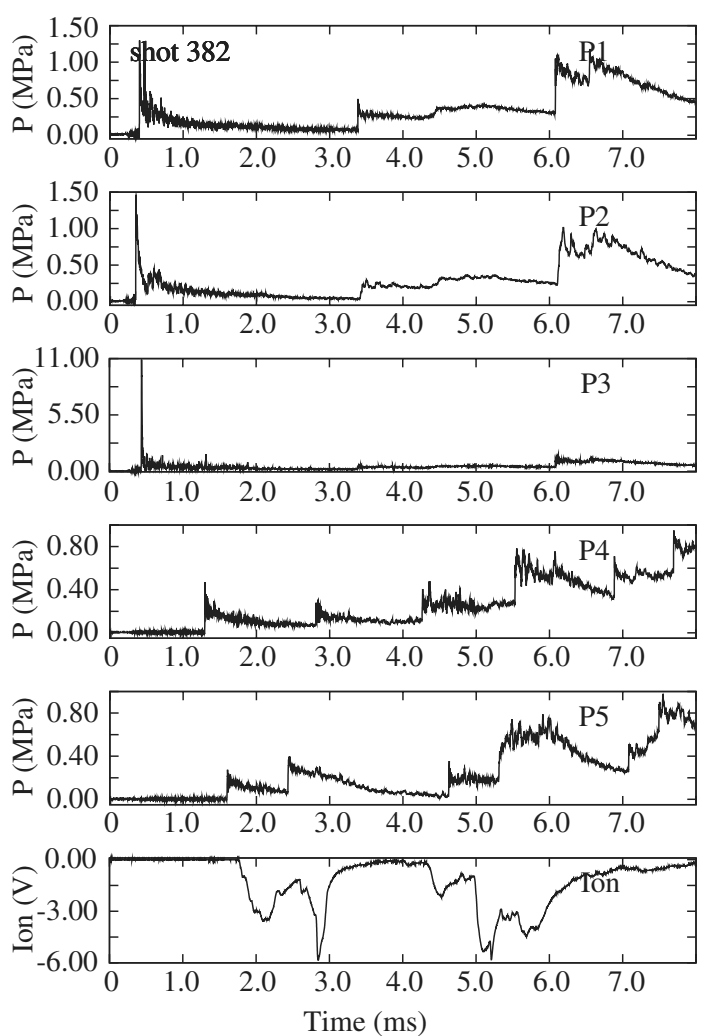

Fig. 27 Pressure and ionization traces from a typical shock initiation experiment. Test-section mixture was stoichiometric ethylene air at 0.10 MPa initial pressure. Traces are labeled and correspond to locations shown in Fig. 28. 


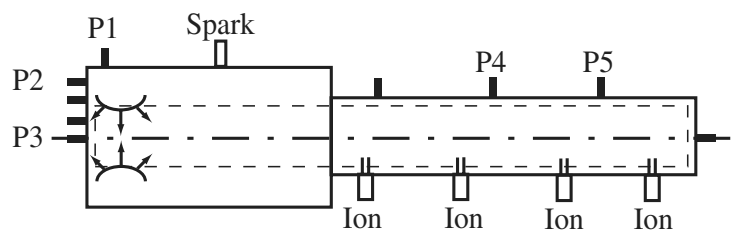

Fig. 28 A schematic of the experimental setup used for attempted initiation of HC-air mixtures using an imploding shock wave.

the wave arrival in the experiments. The nominal wave velocities shown in the previous sections were calculated by dividing the distance $L$ between each transducer by the difference in arrival times $\Delta t$ at each transducer, such that $v_{\text {nom }}=L / \Delta t$. This calculation assumes that the wave was centered on each transducer at the instant of data sampling.

In reality, this was likely not the case. Assuming that the transducer detects pressure changes over its entire head of diameter $d$, a wave detected at the trailing edge of the first transducer and at the leading edge of the second transducer has an actual velocity of

$$
v_{\min }=\frac{L-d}{\Delta t}
$$

but would be calculated to be traveling at $v_{\text {nom }}$. This is the minimum wave velocity that could be mistaken for $v_{\text {nom }}$. Alternatively, a wave detected at the leading edge of the first transducer and at the trailing edge of the second transducer has an actual velocity of

$$
v_{\max }=\frac{L+d}{\Delta t}
$$

but would also be calculated to have a velocity of $v_{\text {nom }}$. Thus, the range of values as a percent of $v_{\text {nom }}$ is

$$
\frac{v_{\max }-v_{\min }}{v_{\text {nom }}} \times 100 \%=\frac{2 d}{L} \times 100 \%
$$

where $d$ was $5.54 \mathrm{~mm}$ and $L$ was $0.19 \mathrm{~m}$ in the test section of these experiments, leading to a maximum possible velocity uncertainty range of $5.8 \%$. This uncertainty was assumed in all velocities derived from transducer measurements. In reality, this is a conservative estimate as waves in this study traveled slower than $2 \mathrm{~mm} / \mu \mathrm{s}$, while the data sampling rate in the test section was $1 \mathrm{MHz}$. Thus, it was unlikely that the wave would even travel across half of the transducer head before being detected.

In the imaging experiments, the camera was triggered by the passage of the wave over a transducer located at the exit of the initiator channels. The signal from the transducer was monitored by a delay generator, which detected the arrival of the wave and triggered the CCD camera. The maximum uncertainty associated with the delay and imaging system was less than $50 \mathrm{~ns}$, thus the uncertainty associated with the imaging times is $\pm 50 \mathrm{~ns}$.

It is also possible to estimate the uncertainty in the initial mixtures used in the experiments. The maximum leak rate of the experiment under vacuum was measured to be $20 \mathrm{~Pa} / \mathrm{min}$. After evacuation of the experiment, $5 \mathrm{~min}$ was required to fill the vessel. Thus, a maximum air contamination of $100 \mathrm{~Pa}$ was possible during the filling procedure. The experiment room was maintained at $295 \pm 3 \mathrm{~K}$. Assuming a worst case compounding of uncertainty for the leak rates and temperature fluctuations, the uncertainty in the wave speed $U_{\mathrm{CJ}}$ as calculated by STANJAN [36] does not exceed $\pm 2 \mathrm{~m} / \mathrm{s}$ corresponding to $0.1 \%$ of the velocity.

\section{Estimating the Effectiveness of the Toroidal Initiator}

In this section, the effectiveness of the toroidal initiator will be compared to that of an initiator tube. Both the amount of gas used and the chemical energy released to create the implosion will be used as figures of merit in the comparison.
Table 6 Critical values for a model initiator tube compared to the toroidal initiator

\begin{tabular}{lcc}
\hline \hline & Toroidal initiator & Tube \\
\hline Energy & $3.62 \mathrm{~kJ}$ & $3.14 \mathrm{~kJ}$ \\
Mass $\mathrm{C}_{2} \mathrm{H}_{2}$ & $0.23 \mathrm{~g}$ & $0.16 \mathrm{~g}$ \\
${\text { Mass } \mathrm{O}_{2}}^{2}$ & $0.28 \mathrm{~g}$ & $0.49 \mathrm{~g}$ \\
\hline
\end{tabular}

\section{Gas Usage of a Typical Initiator Tube}

Little data are available for predicting the necessary initiator tube dimensions for equimolar acetylene-oxygen mixtures, but recent work by Murray et al. [8] has produced a model for stoichiometric acetylene-oxygen initiator mixtures that predicts the necessary initiator tube dimensions based on an extensive data set. The model predicts that for an initiator tube diameter to main tube diameter ratio of $d_{d} / d=0.5$, the initiator tube length $L_{d}$ necessary to initiate a stoichiometric propane-air test-section mixture with a cell size of $\lambda=50 \mathrm{~mm}$ (2 in.) is approximately $L_{d} / \lambda=9$ or $L_{d}=45.7 \mathrm{~cm}$ (18 in.). For a main tube diameter of $d=76 \mathrm{~mm}$ ( 3 in.) used in the toroidal initiator experiments, this corresponds to an initiator tube volume of

$$
\begin{aligned}
& V_{d}=\frac{\pi d^{2}}{4} L_{d} \\
& =\frac{\pi(0.75 \lambda)^{2}}{4} 9 \lambda
\end{aligned}
$$

$$
\approx 4 \lambda^{3}
$$

$$
=524 \mathrm{cc} \text { or } 32 \mathrm{in}^{3}
$$

As previously determined, the toroidal initiator requires $434 \mathrm{cc}$ of equimolar acetylene-oxygen gas to detonate propane air. The required mass of each gas is shown in Table $\underline{6}$.

\section{Energy Input to the Toroidal Initiator}

In the toroidal initiator, the implosion was created by detonating an equimolar acetylene-oxygen mixture. In calculating the input energy $E_{\text {inp }}$ to the toroidal initiator, the chemical energy released is assumed to be the dominant contribution to the initiation process and the energy input from mass flow will be neglected.

The $434 \mathrm{cc}\left(26.5\right.$ in. $\left.^{3}\right)$ volume of equimolar acetylene-oxygen gas used by the toroidal initiator was initially at a temperature of $295 \mathrm{~K}$ and a pressure of $0.10 \mathrm{MPa}$ to initiate a detonation in a tube filled with stoichiometric propane air. The effective heat of reaction $\Delta h^{0}$ of the initiator gas mixture can be approximated in the following fashion [37].

The heat of reaction $\Delta h^{0}$ is defined as the difference in enthalpy of the gas, extrapolated from absolute zero temperature

$$
\begin{aligned}
& h_{1}=h^{01}+C_{p 1} T_{1} \\
& h_{2}=h^{02}+C_{p 2} T_{2} \\
& h^{01}-h^{02}=\Delta h^{0}
\end{aligned}
$$

where the subscript 1 denotes the initial gas state and subscript 2 is the state which has been processed by the detonation wave.

Applying the above relations to the shock-jump condition for energy

$$
h_{1}+\frac{1}{2} w_{1}^{2}=h_{2}+\frac{1}{2} w_{2}^{2}
$$


yields

$$
\Delta h^{0}+C_{p 1} T_{1}+\frac{1}{2} w_{1}^{2}=C_{p 2} T_{2}+\frac{1}{2} w_{2}^{2}
$$

Substituting

$$
P v=R T
$$

and

$$
C_{p}=\frac{\gamma}{\gamma-1} R
$$

into Eq. (22) and rearranging terms, the energy shock-jump condition for a perfect gas is obtained

$$
\begin{gathered}
\Delta h^{0}+R_{1} T_{1}\left(\frac{\gamma_{1}}{\gamma_{1}-1}\right)\left(1+\frac{\gamma_{1}-1}{2} M_{1}^{2}\right) \\
=R_{2} T_{2}\left(\frac{\gamma_{2}}{\gamma_{2}-1}\right)\left(1+\frac{\gamma_{2}-1}{2} M_{2}^{2}\right)
\end{gathered}
$$

Evaluating the postdetonation flow at the CJ surface will set $M_{2}=1$. Solving for $\Delta h^{0}$, the heat of reaction is

$$
\begin{aligned}
\Delta h^{0} & =R_{\mathrm{CJ}} T_{\mathrm{CJ}}\left(\frac{\gamma_{\mathrm{CJ}}}{\gamma_{\mathrm{CJ}}-1}\right)\left(1+\frac{\gamma_{\mathrm{CJ}}-1}{2}\right)-R_{1} T_{1}\left(\frac{\gamma_{1}}{\gamma_{1}-1}\right) \\
& \times\left(1+\frac{\gamma_{1}-1}{2} M_{\mathrm{CJ}}^{2}\right)
\end{aligned}
$$

In this perfect gas, $2-\gamma$, the CJ detonation model, $\gamma_{1}$ and $\gamma_{\mathrm{CJ}}$ are the ratios of specific heats of the gas at the initial state and at the CJ surface, respectively. $T_{\mathrm{CJ}}$ is the temperature at the $\mathrm{CJ}$ surface and $M_{\mathrm{CJ}}$ is the Mach number of the $\mathrm{CJ}$ detonation wave.

STANJAN [36] was used to perform the equilibrium calculations necessary to obtain the CJ parameters. For acetylene-oxygen mixtures, the effective heat of reaction is shown in Fig. 29 as a function of equivalence ratio $\phi$. For the equimolar $(\phi=2.5)$ mixtures used in the toroidal initiator, the effective heat of reaction was determined to be $\Delta h^{0}=7.07 \mathrm{MJ} / \mathrm{kg}$ of the initiator mixture. Thus, the energy released by detonation of the initiator gas mixture for propane air was found to be

$$
E_{\text {toroidal }}=\Delta h^{0} \rho_{1} V_{\text {toroidal }}=3.62 \mathrm{~kJ}
$$

Because the Murray et al. model assumes a stoichiometric acetyleneoxygen initiator gas, the effective heat of reaction of the mixture is found to be $\Delta h^{0}=4.85 \mathrm{MJ}$. This corresponds to an energy release of

$$
E_{d}=\Delta h^{0} \rho_{1} V_{d}=3.14 \mathrm{~kJ}
$$

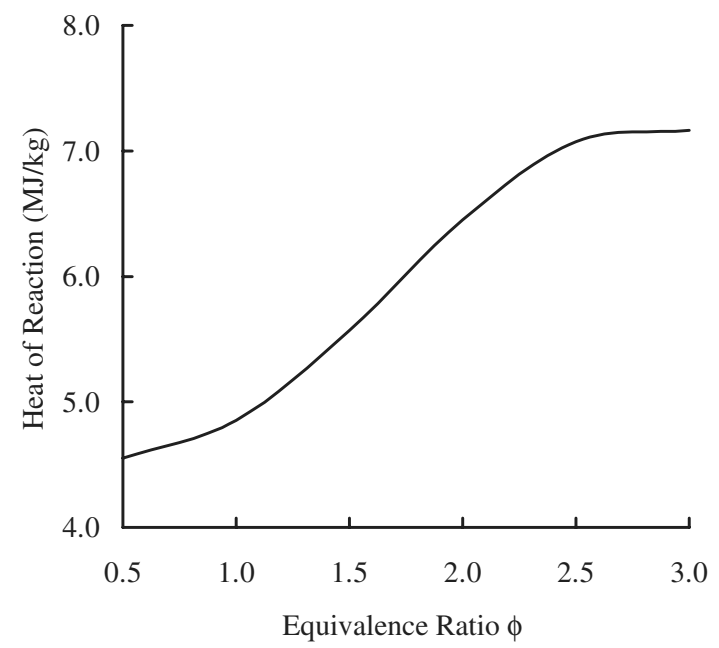

Fig. 29 Effective heat of the reaction of acetylene-oxygen mixtures as a function of the equivalence ratio.
The amount of oxygen necessary for successful initiation is important in performance modeling for PDEs as oxygen tanks will result in payload losses. The masses of fuel and oxygen initiator gas were also calculated and are shown in Table 6. Examination of the amount of initiator gas used by each initiator reveals that the toroidal initiator uses more fuel, but about half the amount of oxygen used by the model initiator tube.

Note that use of an acetylene-oxygen mixture on an actual engine would require storage systems for the acetylene-oxygen gas, decreasing the efficiency of the system. The initiator mixture used in this study was chosen only because it was part of an existing dynamic injection system [29]. In practice, the possibility of replacing the acetylene used for the initiation with the fuel for the primary tube could be explored to eliminate the need for acetylene storage systems.

Designers of PDEs should note that the primary interest of this work was to evaluate the effectiveness of the imploding detonation scheme as compared to DDT. Further effort would be required to evaluate the dynamic initiator as a possible PDE initiator. Although attempts were made during the design of these prototypes to minimize the channel volume of the toroidal initiator, a systematic optimization of its gas usage was not performed. Thus, it may be using more gas than is required to create the imploding waves. Initiator tubes, on the other hand, have undergone significant optimization analyses $[7,8]$ to better understand the minimum amount of gas required for detonation initiation.

\section{Summary and Conclusions}

A detonation initiation device has been presented that generates an imploding toroidal detonation wave using an array of small channels filled with acetylene-oxygen gas and ignited with a single weak spark. The small channels are incorporated inside the wall of the detonation tube to minimize both the size of the initiator and its effect on the flow in the detonation tube. The imploding wave generated with this device is able to initiate detonations in a tube filled with hydrocarbon-oxygen-nitrogen mixtures, including stoichiometric propane air and ethylene air. The minimum volume of acetyleneoxygen gas required to detonate these hydrocarbon-air mixtures was found to exceed the volume of the initiator channels and required overfilling of the initiator, resulting in some gas spillage into the main tube. This overfill volume was found to increase as the sensitivity of the mixture to be detonated decreased. Proximity of the end flange to the wave focus was found to reduce the critical overfill volume by creating symmetry and providing surfaces for wave reflection. An analysis of the effectiveness of the toroidal initiator estimated that it used $13 \%$ more chemical energy than a model initiator tube [19] to detonate propane-air mixtures. The same analysis determined that the toroidal initiator used less oxygen but more hydrocarbon fuel than a model initiator tube. Optimization of the gas usage of the initiator was not performed in this study and may result in increased initiator effectiveness.

An existing approximate solution [12] for cylindrical and spherical imploding waves was adapted to the imploding toroidal geometry. Analysis of the imploding geometry established that the wave diffracts for the first half of the toroidal implosion process, while focusing occurs during the last half. This diffraction is not present in the cylindrical and spherical imploding geometries. In the approximate solution of the toroidal imploding wave, it was assumed that the wave remained at the $\mathrm{CJ}$ velocity during the period of diffraction, as experimental investigations [31] have shown that diffraction will not significantly decay a wave that is sufficiently wide relative to its cell size. This assumption was also supported by experimental observations of the toroidal imploding wave in this study, which showed that only a small amount of diffraction occurred.

Imploding toroidal waves in ethylene-oxygen mixtures were observed to implode at a steady velocity $9 \%$ lower than the CJ velocity of the mixture. Pressure measurements of the imploding wave taken off the axis of symmetry were above the values of the approximate solution early in the diffraction process due to the 
presence of regular reflection and Mach stems at the pressure-sensing wall. A nonreacting numerical simulation of an imploding shock wave illustrated the reflection modes present at the wall and the postshock pressure evolution of an imploding toroidal shock wave. Pressures measured at the focal axis ranged from 6 to 10 times $P_{\mathrm{CJ}}$.

The results are of interest to designers of pulse detonation engines in that they indicate that the locally high pressures and temperatures associated with the toroidal implosion did not increase the effectiveness of the toroidal initiator above that of a typical initiator tube. The study also provides experimental measurements and an approximate solution for toroidal imploding detonation waves, which were not previously available. Future work will focus on the ability of imploding shocks rather than detonations to initiate hydrocarbon-oxygen-nitrogen mixtures.

\section{Acknowledgments}

This work was sponsored by the Office of Naval Research Grants "Pulse Detonation Engines: Initiation, Propagation and Performance" and "Multidisciplinary Study of Pulse Detonation Engine," by General Electric, and by the Department of Defense and Army Research Office through a National Defense Science and Engineering Graduate Fellowship. The authors are grateful for the assistance of M. Grunthaner, J. Haggerty, B. St. John, and F. Pintgen for their assistance with the design and testing of the toroidal initiator and to $\mathrm{H}$. Hornung for his assistance with the numerical simulation. The authors are also grateful to N. Nebeker and P. Nagel at Cordin Scientific Imaging for use of their Model 220 CCD camera.

\section{References}

[1] Roy, G., Frolov, S., Borisov, A., and Netzer, D., "Pulse Detonation Propulsion: Challenges, Current Status and Future Perspective," Progress in Energy and Combustion Science, Vol. 30, No. 6, 2004, pp. 545-672.

[2] Brophy, C., Werner, S., and Sinibaldi, J., "Performance Characterization of a Valveless Pulse Detonation Engine," AIAA Paper 20031344, Jan. 2003.

[3] Schauer, F., Miser, C., Tucker, K., Bradley, R., and Hoke, J., "Detonation Initiation of Hydrocarbon-Air Mixtures in a Pulse Detonation Engine," AIAA Paper 2005-1343, Jan. 2005.

[4] Urtiew, P., and Oppenheim, A., "Experimental Observations of the Transition to Detonation in an Explosive Gas," Proceedings of the Royal Society of London A, Vol. 295, No. 1440, 1966, pp. 13-28.

[5] Higgins, A., Yoshinaka, P., and Lee, J., "Sensitization of Fuel-Air Mixtures for Deflagration to Detonation Transition," Proceedings of the International Colloquium on Control of Detonation Processes, Elex KM, Moscow, 2000

[6] Cooper, M., Jackson, S., Austin, J., Wintenberger, E., and Shepherd, J. E., "Direct Experimental Impulse Measurements for Detonations and Deflagrations," Journal of Propulsion and Power, Vol. 18, No. 5, 2002, pp. 1033-1041.

[7] Kuznetsov, M., Dorofeev, S., Efimenko, A., Alekseev, V., and Breitung, W., "Experimental and Numerical Studies on Transmission of Gaseous Detonation to a Less Sensitive Mixture," Shock Waves, Vol. 7, No. 5, 1997, pp. 297-304.

[8] Murray, S., Zhang, D., and Gerrard, K., "Critical Parameters for Pulse Detonation Engine Pre-Detonator Tubes," International Colloquium on the Dynamics of Explosions and Reactive Systems, Hakone, Japan, 27 July-1 Aug. 2003.

[9] Brophy, C., Sinibaldi, J., and Damphousse, P., "Initiator Performance for Liquid-Fueled Pulse Detonation Engines," AIAA Paper 02-0472, Jan. 2002.

[10] Jackson, S., Grunthaner, M., and Shepherd, J., "Wave Implosion as an Initiation Mechanism for Pulse Detonation Engines," AIAA Paper 2003-4280, July 2006.

[11] Whitham, G., "On the Propagation of Shock Waves Through Regions of Non-Uniform Area or Flow," Journal of Fluid Mechanics, Vol. 4, No. 4, 1958, pp. 337-360.

[12] Lee, J., and Lee, B., "Cylindrical Imploding Shock Waves," Physics of Fluids, Vol. 8, No. 12, 1965, pp. 2148-2152.

[13] Jiang, Z., and Takayama, K., "Reflection and Focusing of Toroidal Shock Waves from Coaxial Annular Shock Tubes," Computers and Fluids, Vol. 27, No. 5-6, 1998, pp. 553-562.
[14] Takayama, K., Kleine, H., and Gronig, H., "An Experimental Investigation of the Stability of Converging Cylindrical Shock Waves in Air," Experiments in Fluids, Vol. 5, No. 5, 1987, pp. 315-322.

[15] Devore, C., and Oran, E., "The Stability of Imploding Detonations in the Geometrical Shock Dynamics (CCW) Model," Physics of Fluids A, Vol. 4, No. 4, 1992, pp. 835-844.

[16] Oran, E., and Devore, C., "The Stability of Imploding DetonationsResults of Numerical Simulations," Physics of Fluids, Vol. 6, No. 1, 1994, pp. 369-380.

[17] Terao, K., Akaba, H., and Shiraishi, H., "Spherically Imploding Detonation Waves Initiated by 2-Step Divergent Detonation," Shock Waves, Vol. 4, No. 4, 1995, pp. 187-193.

[18] Akbar, R., "Mach Reflection of Gaseous Detonations," Ph.D. Thesis, Rensselaer Polytechnic Institute, Troy, NY, 1997.

[19] Murray, S., Thibault, P., Zhang, F., Bjerketvedt, D., Sulmistras, A., Thomas, G., Jenssen, A., and Moen, I., "The Role of Energy Distribution on the Transmission of Detonation," Proceedings of the International Colloquium on Control of Detonation Processes, Elex KM, Moscow, 2000.

[20] Jackson, S., and Shepherd, J., "Initiation Systems for Pulse Detonation Engines," AIAA Paper 2002-3627, July 2002.

[21] Jackson, S., "Gaseous Detonation Initiation via Wave Implosion," Ph.D. Thesis, California Institute of Technology, Pasadena, CA, 2005.

[22] Li, C., and Kailasanath, K., "Detonation Initiation by Annular-JetInduced Imploding Shocks," Journal of Propulsion and Power, Vol. 21, No. 1, 2005, pp. 183-186.

[23] Jackson, S., and Shepherd, J., "Detonation Initiation via Imploding Shock Waves," AIAA Paper 2004-3919, July 2004.

[24] Yu, S.-T. J., Wang, B., and He, H., "Direct Calculation of Wave Implosion for Detonation Initiation in Pulsed Detonation Engines," Proceedings of the Seventeenth ONR Propulsion Meeting, edited by G. Roy, and A. Ghoniem, Office of Naval Research, Arlington, June 2004, pp. 169-180.

[25] Wang, B., He, H., and Yu, S.-T., "Direct Calculation of Wave Implosion for Detonation Initiation in Pulsed Detonation Engines," AIAA Paper 2005-1306, Jan. 2005.

[26] de Witt, B., Ciccarelli, G., Zhang, F., and Murray, S., "Shock Reflection Detonation Initiation Studies for Pulse Detonation Engines," AIAA Paper 2004-3747, July 2004.

[27] Jackson, S., Austin, J., and Shepherd, J., "Planar Detonation Wave Initiation in Large-Aspect-Ratio Channels," AIAA Journal, Vol. 44, No. 10, 2006, pp. 2422-2425.

[28] Grunthaner, M., Jackson, S., and Shepherd, J., "Design and Construction of an Annular Detonation Initiator," GALCIT TR 2001005, Graduate Aeronautical Laboratories, California Institute of Technology, Pasadena, CA, 2001.

[29] Austin, J., "The Role of Instability in Gaseous Detonation," Ph.D. Thesis, California Institute of Technology, Pasadena, CA, 2003.

[30] Schultz, E., and Shepherd, J., "Validation of Detailed Reaction Mechanisms for Detonation Simulation," GALCIT TR FM99-5, Graduate Aeronautical Laboratories, California Institute of Technology, Pasadena, CA, 2000.

[31] Benedick, W., Knystautas, R., and Lee, J., "Large Scale Experiments on the Transmission of Fuel-Air Detonations from Two-Dimensional Channels," Dynamics of Detonations and Explosions, Vol. 94, Progress in Astronautics and Aeronautics, AIAA, New York, 1984, pp. 546-555.

[32] Quirk, J., "AMRITA-A Computational Facility (for CFD Modelling)," VKI 29th CFD Lecture Series, ISSN 0377-8312.

[33] Nettleton, M. A., Gaseous Detonations, Chapman and Hall, London, 1987.

[34] Meltzer, J., Shepherd, J. E., Akbar, R., and Sabet, A., "Mach Reflection of Detonation Waves," Dynamics of Detonations and Explosions, Vol. 153, Progress in Astronautics and Aeronautics, AIAA, New York, 1993, pp. 78-94.

[35] Li, C., and Kailasanath, K., "Detonation Initiation in Pulse Detonation Engines," AIAA Paper 2003-1170, Jan. 2003.

[36] Reynolds, W. C., "The Element Potential Method for Chemical Equilibrium Analysis: Implementation in the Interactive Program STANJAN, Version 3," TR, Department of Mechanical Engineering, Stanford University, Stanford, CA, 1986.

[37] Thompson, P., Compressible-Fluid Dynamics, McGraw-Hill, New York, 1988. 